\title{
Stigma and intersectionality: a systematic review of systematic reviews across HIV/ AIDS, mental illness, and physical disability
}

\author{
Fatimah Jackson-Best ${ }^{1 *}$ (D) and Nancy Edwards ${ }^{2}$
}

\begin{abstract}
Background: Stigma across HIV/AIDS, mental illness, and physical disability can be co-occurring and may interact with other forms of stigma related to social identities like race, gender, and sexuality. Stigma is especially problematic for people living with these conditions because it can create barriers to accessing necessary social and structural supports, which can intensify their experiences with stigma. This review aims to contribute to the knowledge on stigma by advancing a cross-analysis of HIV/AIDS, mental illness, and physical disability stigma, and exploring whether and how intersectionality frameworks have been used in the systematic reviews of stigma.

Methods: A search of the literature was conducted to identify systematic reviews which investigated stigma for HIV/ AIDS, mental illness and/or physical disability. The electronic databases MEDLINE, CINAHL, EMBASE, COCHRANE, and PsycINFO were searched for reviews published between 2005 and 2017. Data were extracted from eligible reviews on: type of systematic review and number of primary studies included in the review, study design study population(s), type(s) of stigma addressed, and destigmatizing interventions used. A keyword search was also done using the terms "intersectionality", "intersectional", and "intersection"; related definitions and descriptions were extracted. Matrices were used to compare the characteristics of reviews and their application of intersectional approaches across the three health conditions.
\end{abstract}

Results: Ninety-eight reviews met the inclusion criteria. The majority (99\%) of reviews examined only one of the health conditions. Just three reviews focused on physical disability. Most reviews (94\%) reported a predominance of behavioural rather than structural interventions targeting stigma in the primary studies. Only 17\% of reviews used the concept and/or approach of intersectionality; all but one of these reviews examined HIV/AIDS.

Conclusions: The lack of systematic reviews comparing stigma across mental illness, HIV/AIDS, and physical disability indicates the need for more cross-comparative analyses among these conditions. The integration of intersectional approaches would deepen interrogations of co-occurring social identities and stigma.

Keywords: Stigma, Intersectionality, HIV/AIDS, Mental illness, Physical disability

\section{Background}

Stigma is a dynamic process enacted through structures and individuals, and mediated by relationships of power, control, and domination that are continuously produced and reproduced by actors [1]. At its foundation, stigma is about social inequality and social control, which create a hierarchy that devalues stigmatized people [1].

\footnotetext{
* Correspondence: fatimahzjackson@gmail.com

'I Am One, 58C Cypress Ridge, Union Hall, San Fernando, Trinidad and Tobago

Full list of author information is available at the end of the article
}

Stigma is especially problematic for people living with HIV/AIDS (Human Immunodeficiency Virus Infection and Acquired Immune Deficiency Syndrome), mental illness, and physical disabilities because it can create barriers to accessing health care, education, employment, and affordable housing, which in turn, may exacerbate the experience of marginalization [2, 3]. Furthermore, people often live with more than one of these health conditions and may simultaneously experience different kinds of health-related stigma. For example, research indicates that people living with HIV/AIDS have higher

(C) The Author(s). 2018 Open Access This article is distributed under the terms of the Creative Commons Attribution 4.0 International License (http://creativecommons.org/licenses/by/4.0/), which permits unrestricted use, distribution, and 
rates of depression and anxiety in comparison to the general population [4], and people with physical disabilities are at an elevated risk for depressive symptoms and major depressive disorder [5].

The overlap of different kinds of disease stigma and the rooted-ness of stigma in larger systems of inequality and webs of power have pushed researchers to consider different ways to investigate and analyze it. Given stigma's links to historical and contemporary manifestations of inequality, power, and systems of domination; intersectionality offers a promising theoretical approach to examine research on stigma. Black feminists, who coined and produced theory on the concept of intersectionality, highlighted how multiple oppression and structural inequalities exist in matrices of domination, which in turn, reinforce unequal relationships of power amongst people; and between people and social institutions such as healthcare, housing, and the law [6-8]. Originally used in feminist theory to describe Black women's positions within webs of power, intersectionality has been taken up by health sciences researchers to help deepen their analyses of structural and systemic issues in health, and the inequalities and inequities they create $[9,10]$.

This review of reviews seeks to contribute to the knowledge on stigma by advancing a cross-analysis of HIV/ AIDS, mental illness, and physical disability stigma, and exploring whether and how intersectionality frameworks have been used in the systematic reviews of stigma.

\section{Methods}

We adapted Arskey and O'Malley's [11] scoping review framework to guide the methodology of our review. We used the same subheadings as the authors for the "identifying the research questions" [11] and "identifying relevant studies" [11] stages. However, 'relevance review' was used instead of "study selection" [11], and we collapsed "charting the data" and "collating, summarizing and reporting the results" [11] into a single subheading called 'data extraction, collation and analysis' to reflect our methodology.

\section{Identifying the research question(s)}

The research questions guiding our review were:

1. What are the characteristics of systematic reviews examining sources of and influences on stigma among those living with HIV/AIDS, mental illness, and/or physical disability?

2. Has intersectionality been used and how has it been used in systematic reviews of stigma and stigma reduction interventions for those living with HIV/ AIDS, mental illness, and/or physical disability?

\section{Identifying relevant studies}

The search strategy and electronic database searches were developed and conducted with the assistance of a librarian at the University of Ottawa Health Sciences Library. Electronic databases that publish health-related research and information were accessed. Five databases were searched: MEDLINE, Cumulative Index to Nursing and Allied Health Literature (CINAHL), EMBASE, COCHRANE (Database of Systematic Reviews, EBM Reviews- ACP Journal Club, and EBM Reviews- Database of Abstracts of Reviews of Effects), and PsycINFO.

Several databases indicated that prior to the year 2005, stigma was not a mesh heading, and terms such as "discrimination", "stereotyping", and "prejudice" were commonly used. Although we were searching for publications from 2005 and later, we used both the newer and older search terms to ensure that we captured all relevant reviews. The search headings used in all five electronic databases included: "stigma", OR "prejudice", OR "social discrimination", OR "social stigma", OR "stereotyping", OR "stereotyped attitudes", OR "shame". Keywords used in the search of the databases also included truncated versions of the following terms: discrimination stigma, and prejudice.

Search filters (hedges) were used in the MEDLINE, EMBASE, and PsycINFO databases to limit retrievals to systematic reviews. These filters were not necessary for the COCHRANE database because it only publishes systematic reviews. A filter to restrict retrieved papers to systematic reviews was also applied to the CINAHL database search.

Inclusion criteria for the database searches were:

1. Reviews written in English language

2. Reviews published between January 2005 and November 2017 (inclusive).

Exclusion criteria for the database searches were:

\section{Dissertations.}

Zotero, a software reference package, was used to manage the citations.

\section{Relevance review}

Following the first database search, both authors independently reviewed a sample $(n=15)$ of retrieved titles and abstracts for relevance. They then met to discuss discrepancies in their assessments, and refine the final inclusion criteria for reviews, which were:

- Systematic reviews using qualitative, quantitative, or mixed methods; 
- Focus on health-related stigma experienced by study populations with HIV/AIDS, mental illness, and/or physical disability. The definition of mental illness used in the review is consistent with the following definition: "a spectrum of cognitions, emotions and behaviours that interfere with interpersonal relationships as well as functions required for work, at home and in school" [2]. The definition of physical disability used in the review is consistent with the following definition: "any infirmity, malformation or disfigurement that is caused by bodily injury, birth defect or illness and, without limiting the generality of the foregoing, includes diabetes mellitus, epilepsy, a brain injury, any degree of paralysis, amputation, lack of physical co-ordination, blindness or visual impediment, deafness or hearing impediment, muteness or speech impediment, or physical reliance on a guide dog or other animal or on a wheelchair or other remedial appliance or device" [12]

- Stigma is an outcome, result and/or theme of the review and discussed in the research findings; and,

- Review includes measurement tools; conceptual frameworks and theoretical frameworks such as, but not limited to intersectionality; guidance documents; and/or methodological approaches for exploring stigma.

The titles and abstracts of all citations were then screened for relevance by the authors. When relevance could not be ascertained, the full paper was retrieved and reviewed to make a relevance decision.

\section{Data extraction, collation and analysis}

Data were extracted from the reviews using the following categories: aim/objective, specific health issue addressed (i.e. type of mental illness or disability), type of systematic review and number of primary studies included in the review, their geographic location, study design (qualitative, quantitative or mixed methods), study population, type(s) of stigma addressed (interpersonal stigma, intrapersonal stigma, and structural/institutional stigma), and destigmatizing interventions used. We also extracted key findings and recommendations from each review. Data were entered into a table in Microsoft Excel. To ensure that we captured all descriptors of intersectionality, we then did a keyword search of each eligible review paper using the terms "intersectionality", "intersectional", and "intersection". We extracted all definitions and descriptions of these terms from these papers as well as any related findings. We used matrices to compare the characteristics of reviews and their application of intersectional approaches across the three health conditions.

\section{Results}

The electronic database search yielded 2405 citations. In the first exclusion phase, 691 duplicates were eliminated leaving 1714 citations for relevance review. In the second exclusion phase, 1487 papers were eliminated because they were ineligible or found to be additional duplicates. In total, 227 papers were identified for a full text review. One hundred twenty-nine papers were found to be ineligible during the third exclusion phase. In total, 98 retrieved systematic reviews of stigma were included in our review (See Fig. 1 for an overview of the search results, and Table 1 for an overview of the reviews).

\section{Characteristics of the systematic reviews Types of systematic reviews included in the review}

In total, eight types of reviews were found (Table 2). The most frequent types were integrative reviews $(38 \%, n=$ 37), followed by quantitative systematic reviews with no assessment of methodological quality $(17 \%, n=17)$, meta-analysis $(20 \%, n=20)$, and quantitative systematic reviews with an assessment of methodological quality $(19 \%, n=19)$. There were fewer than five reviews for each of the following categories: meta-ethnography $(4 \%, n=4)$, qualitative systematic reviews with an assessment of methodological quality $(4 \%, n=4)$, qualitative systematic reviews with no assessment of methodological quality $(1 \%, n=1)$, critical synthesis $(1 \%, n=1)$, scoping review $(1 \%, n=1)$, meta-study $(1 \%, n=1)$, and meta-ethnography combined with mixed methods review $(1 \%, n=1)$.

\section{Disease/condition focus, publication date, and geographic location in the primary studies}

Primary studies were reported from over 60 countries; all continents were represented except Antarctica. The majority of the reviews were disease specific, with the largest proportion found for mental illnesses $(61 \%, n=$ $60)$, followed by HIV/AIDS (34\%, $n=34)$; a smaller number of reviews were found for physical disability stigma $(3 \%, n=3)$. A single review $(1 \%, n=1)$ looked at stigma across all three health conditions [13] and included other stigmatized health conditions including leprosy, tuberculosis, and epilepsy.

Most reviews of HIV/AIDS and mental illness stigma had been published within the last 5 years $(64 \%, n=63)$. With only three reviews for physical disability stigma, no publication pattern was discernable.

\section{Sample characteristics of the systematic reviews}

Across the reviews, the study populations were mostly comprised of people living with one of the three health conditions. For example, people living with HIV/AIDS 


Search terms:
Search Headings: "Stigma", OR "prejudice",
OR "social discrimination", OR “social
stigma", OR "stereotyping", OR
"stereotyped attitude", OR "shame", OR
"psychosocial"
Keyword search (truncated): discriminat,
stigma, prejudic
Protocols and filters: hedges, "review",
"systematic review", "meta-analysis"
Dating from 2005-2017, English language
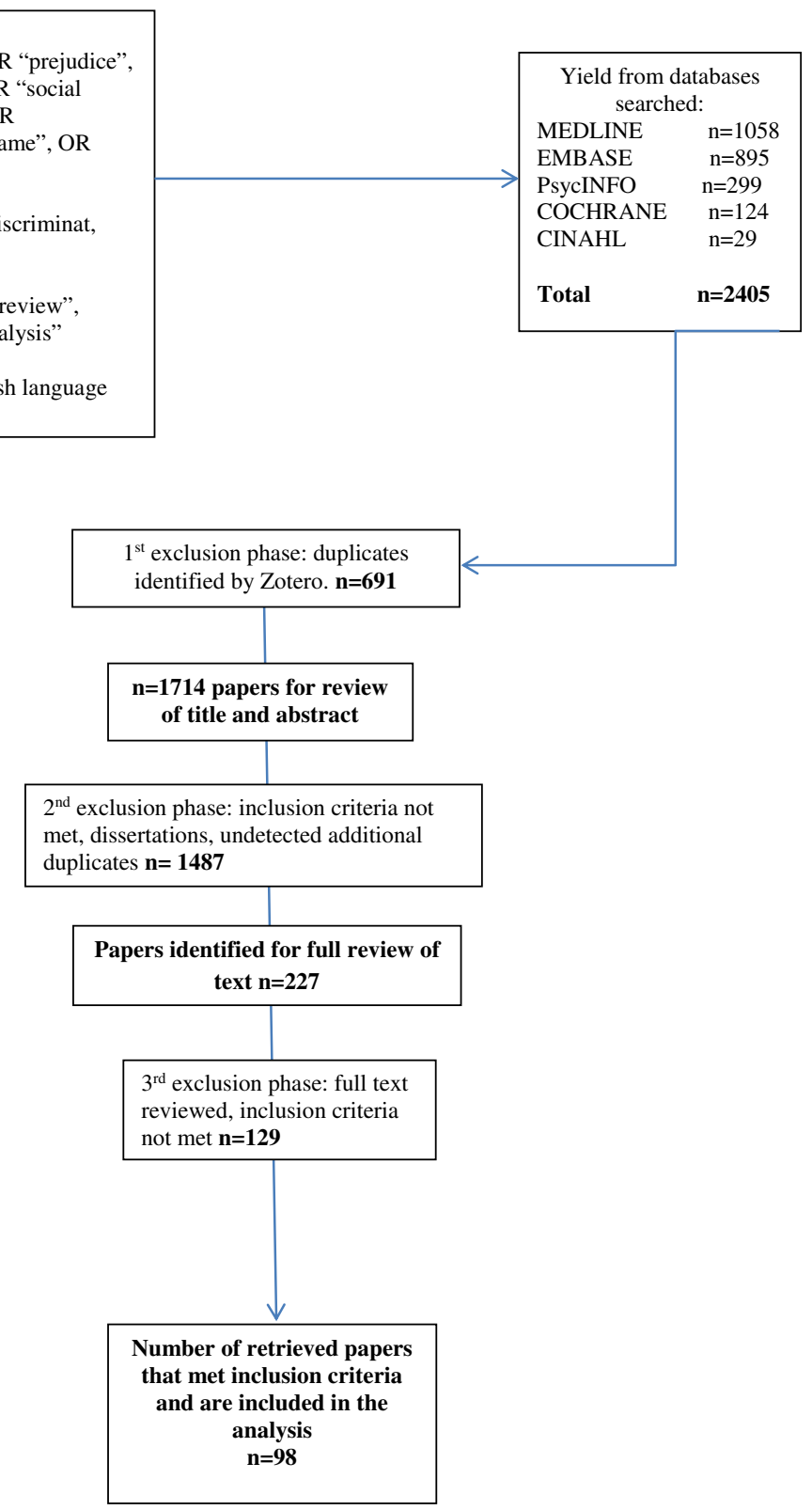

Fig. 1 Search results

(PLWHA) were most commonly included in primary research studies on HIV/AIDS stigma $(37 \%, n=22)$. In the reviews on mental illness stigma, the predominant study populations in the primary studies were people living with mental illness and mental healthcare consumers/ users $(50 \%, n=17)$. There were three reviews in the physical disability stigma category: one examined people living with a physical disability $(33 \%, n=1)$, the second focused on those who interacted with people living with disabilities $(33 \%, n=1)$, and the third explored how disability has been considered in nursing and healthcare literature $(33 \%, n=1)$ [14].

\section{Stigma type and interventions included in the primary research}

Table 3 shows the number and percentages of each type of stigma investigated in the systematic reviews. Nearly half $(47 \%)$ of the reviews discussed more than one stigma type, even when the type of stigma described was not an eligibility criterion. Across the health conditions, various stigma types were examined: $78.5 \%$ examined intrapersonal forms of stigma (i.e. self-stigma, internalized stigma, perceived stigma, affiliate stigma), $48 \%$ of the reviews looked at interpersonal forms of stigma (i.e. social stigma, public stigma, enacted stigma, cultural 
Table 1 Overview of 98 systematic reviews on stigma and HIV/AIDS, mental illness, and physical disability

\begin{tabular}{|c|c|c|c|c|}
\hline Author(s), publication year & Title of paper & $\begin{array}{l}\text { Type(s) of systematic } \\
\text { review }\end{array}$ & $\begin{array}{l}\text { Number of } \\
\text { primary studies } \\
\text { included in the } \\
\text { review }\end{array}$ & $\begin{array}{l}\text { Geographic location(s) } \\
\text { of the primary studies }\end{array}$ \\
\hline \multicolumn{5}{|l|}{ Mental illness } \\
\hline Abiri et al. (2016) [32] & $\begin{array}{l}\text { Stigma related avoidance in people living } \\
\text { with Severe Mental IIIness (SMI): Findings of } \\
\text { an integrative review }\end{array}$ & Integrative review & 21 & $\begin{array}{l}\text { Africa, Asia, Middle East, } \\
\text { Western Europe }\end{array}$ \\
\hline Ali et al. (2017) [33] & $\begin{array}{l}\text { Perceived barriers and facilitators towards } \\
\text { help-seeking for eating disorders: A systematic } \\
\text { review }\end{array}$ & integrative review & 13 & $\begin{array}{l}\text { USA, Australia, Norway, } \\
\text { UK, Germany }\end{array}$ \\
\hline Ando et al. (2013) [34] & $\begin{array}{l}\text { Review of mental-health-related stigma in } \\
\text { Japan }\end{array}$ & integrative Review & 19 & Japan \\
\hline Ando et al. (2011) [35] & $\begin{array}{l}\text { The simulation of hallucinations to reduce the } \\
\text { stigma of schizophrenia: A systematic review }\end{array}$ & meta-ethnography & 11 & USA, Australia, Canada \\
\hline $\begin{array}{l}\text { Angermeyer et al. (2011) } \\
\text { [36] }\end{array}$ & $\begin{array}{l}\text { Biogenetic explanations and public } \\
\text { acceptance of mental illness: Systematic } \\
\text { review of population studies }\end{array}$ & $\begin{array}{l}\text { quantitative } \\
\text { systematic review } \\
\text { with no assessment } \\
\text { of methodological } \\
\text { quality }\end{array}$ & 33 & $\begin{array}{l}\text { North America, Asia, } \\
\text { South America, Africa, } \\
\text { Australia }\end{array}$ \\
\hline Boyd et al. (2014) [37] & $\begin{array}{l}\text { Internalized Stigma of Mental IIIness (ISMI) } \\
\text { scale: A multinational review. }\end{array}$ & $\begin{array}{l}\text { quantitative } \\
\text { systematic review } \\
\text { with no assessment } \\
\text { of methodological } \\
\text { quality }\end{array}$ & 81 & USA \\
\hline Brohan et al. (2010) [38] & $\begin{array}{l}\text { Experiences of mental illness stigma, prejudice } \\
\text { and discrimination: A review of measures }\end{array}$ & $\begin{array}{l}\text { quantitative } \\
\text { systematic review } \\
\text { with assessment of } \\
\text { methodological } \\
\text { quality }\end{array}$ & 57 & Not available \\
\hline $\begin{array}{l}\text { Castaldelli-Maia et al. (2011) } \\
\text { [39] }\end{array}$ & $\begin{array}{l}\text { Perceptions of and attitudes toward } \\
\text { antidepressant }\end{array}$ & $\begin{array}{l}\text { quantitative } \\
\text { systematic review } \\
\text { with no assessment } \\
\text { of methodological } \\
\text { quality }\end{array}$ & 32 & USA, UK \\
\hline Clarke et al. (2014) [40] & $\begin{array}{l}\text { Emergency department staff attitudes towards } \\
\text { mental health consumers: A literature review } \\
\text { and thematic content analysis }\end{array}$ & integrative review & 42 & $\begin{array}{l}\text { USA, UK, Canada, } \\
\text { Australia, Ireland, } \\
\text { Sweden, Finland, China, } \\
\text { New Zealand }\end{array}$ \\
\hline Clement et al. (2015) [41] & $\begin{array}{l}\text { What is the impact of mental health-related } \\
\text { stigma on help-seeking? A systematic review } \\
\text { of quantitative and qualitative studies }\end{array}$ & integrative review & 144 & $\begin{array}{l}\text { USA, Canada, Europe, } \\
\text { Australia and New } \\
\text { Zealand, Asia, South } \\
\text { America }\end{array}$ \\
\hline Clement et al. (2013) [42] & $\begin{array}{l}\text { Mass media interventions for reducing mental } \\
\text { health-related stigma }\end{array}$ & $\begin{array}{l}\text { COCHRANE } \\
\text { quantitative } \\
\text { systematic review } \\
\text { with assessment of } \\
\text { methodological } \\
\text { quality }\end{array}$ & 22 & Not available \\
\hline Corrigan et al. (2015) [43] & $\begin{array}{l}\text { Do the effects of antistigma programs persist } \\
\text { over time? Findings from a meta-analysis }\end{array}$ & meta-analysis & 72 & Not available \\
\hline Corrigan et al. (2012) [44] & $\begin{array}{l}\text { Challenging the public stigma of mental } \\
\text { illness: A meta-analysis of outcome studies }\end{array}$ & meta-analysis & 72 & $\begin{array}{l}\text { Europe, North America, } \\
\text { South America, Asia, } \\
\text { Australia. }\end{array}$ \\
\hline Dalky (2012) [45] & $\begin{array}{l}\text { Mental illness stigma reduction interventions: } \\
\text { Review of intervention trials }\end{array}$ & $\begin{array}{l}\text { quantitative } \\
\text { systematic review } \\
\text { with assessment of } \\
\text { methodological } \\
\text { quality }\end{array}$ & 14 & Not available \\
\hline $\begin{array}{l}\text { de Mendonca Lima \& } \\
\text { Lopes (2012) [46] }\end{array}$ & $\begin{array}{l}\text { Systematic review on origin of stigma and } \\
\text { discrimination against old persons with }\end{array}$ & integrative review & 59 & Not available \\
\hline
\end{tabular}


Table 1 Overview of 98 systematic reviews on stigma and HIV/AIDS, mental illness, and physical disability (Continued)

\begin{tabular}{llll}
\hline Author(s), publication year $\quad$ Title of paper & $\begin{array}{l}\text { Type(s) of systematic } \\
\text { review }\end{array}$ & $\begin{array}{l}\text { Number of } \\
\text { primary studies } \\
\text { included in the } \\
\text { review }\end{array}$
\end{tabular}

mental disorders

Doley et al. (2017) [47]

Interventions to reduce the stigma of eating disorders: A systematic review and metaanalysis

Edwards et al. (2015) [48]

What do we know about the risks for young people moving into, through and out of inpatient mental health care? Findings from an evidence synthesis

Ellison et al. (2013) [49]

Bipolar disorder and stigma: A systematic review of the literature quantitative

systematic review

with no assessment

of methodological

quality and meta-

analysis

integrative review

integrative review

integrative review

so the art in European research reducing social exclusion and stigma related to mental health: A systematic mapping of the literature

Firmin et al. (2016) [51]

Gerlinger et al. (2013) [52]

Griffiths et al. (2014) [53]

Guruge et al. (2017) [18]

Hanisch et al. (2016) [54]

Haugen et al. (2017) [55]

Hawke et al. (2013) [56]

Janouskova et al. (2017) [57]

Jorm (2012) [58]
Stigma resistance is positively associated with psychiatric and psychosocial outcomes: A meta-analysis

Personal stigma in schizophrenia spectrum disorders: a systematic review of prevalence rates, correlates, impact and interventions

Effectiveness of programs for reducing the stigma associated with mental disorders. A meta-analysis of randomized controlled trials

Knowing so much, yet knowing so little: A scoping review of interventions that address the stigma of mental illness in the Canadian context

The effectiveness of interventions targeting the stigma of mental illness at the workplace: A systematic review

Mental health stigma and barriers to mental health care for first responders: A systematic review and meta-analysis

Stigma and bipolar disorder: A review of the literature

Can video interventions be used to effectively destigmatize mental illness among young people? A systematic review

Belief in the dangerousness of people with quantitative systematic review with assessment of methodological quality

quantitative systematic review with assessment of methodological quality and metaanalysis

integrative review

quantitative systematic review with assessment of methodological quality

integrative review
40

18

Australia, US, UK, Indonesia

USA, UK, Finland, Canada, Norway

Public Stigma: UK, Germany, USA, Hong Kong, Malaysia, Canada, Japan, Singapore,

Pakistan. Internalized/ Affiliative stigma: USA, Canada, Australia, Turkey, UK, South America

UK, Finland, Sweden, Germany

Not available

USA, Europe, North America, Australia, Asia

USA, Australia, Hong Kong, Finland, Russia, Turkey

Canada

Europe, US or Canada, Australia, Asia

United States, Ireland, Canada

Not available

US, Europe, China, Australia

Japan, Germany, 
Table 1 Overview of 98 systematic reviews on stigma and HIV/AIDS, mental illness, and physical disability (Continued)

\begin{tabular}{|c|c|c|c|c|}
\hline Author(s), publication year & Title of paper & $\begin{array}{l}\text { Type(s) of systematic } \\
\text { review }\end{array}$ & $\begin{array}{l}\text { Number of } \\
\text { primary studies } \\
\text { included in the } \\
\text { review }\end{array}$ & $\begin{array}{l}\text { Geographic location(s) } \\
\text { of the primary studies }\end{array}$ \\
\hline & mental disorders: A review & & & $\begin{array}{l}\text { Australia, Canada, } \\
\text { Turkey, Spain, Nigeria, } \\
\text { India, Brazil, Singapore, } \\
\text { USA }\end{array}$ \\
\hline $\begin{array}{l}\text { Kaushik et al.\& } \\
\text { Kyriakopoulos (2016) [59] }\end{array}$ & $\begin{array}{l}\text { The stigma of mental illness in children and } \\
\text { adolescents: A systematic review }\end{array}$ & integrative review & 42 & $\begin{array}{l}\text { USA, United Kingdom, } \\
\text { Ireland, Israel, Australia, } \\
\text { Iran, Canada, Greece, } \\
\text { Japan. }\end{array}$ \\
\hline Kvaale et al. (2013) [60] & $\begin{array}{l}\text { Biogenetic explanations and stigma: A meta- } \\
\text { analytic review of associations among } \\
\text { laypeople }\end{array}$ & meta-analysis & 25 & Not available \\
\hline Kvaale et al. (2013) [61] & $\begin{array}{l}\text { The 'side effects' of medicalization: A meta- } \\
\text { analytic review of how biogenetic } \\
\text { explanations affect stigma }\end{array}$ & meta-analysis & 28 & Not available \\
\hline $\begin{array}{l}\text { Livingston \& Boyd. (2010) } \\
\text { [30] }\end{array}$ & $\begin{array}{l}\text { Correlates and consequences of internalized } \\
\text { stigma for people living with mental illness: A } \\
\text { systematic review and meta-analysis }\end{array}$ & meta-analysis & 127 & Not available \\
\hline Mak et al. (2007) [62] & Meta-analysis of stigma and mental health & meta-analysis & 49 & $\begin{array}{l}\text { North America, Europe, } \\
\text { Australia, Asia }\end{array}$ \\
\hline $\begin{array}{l}\text { Malachowski \& Kirsh (2013) } \\
\text { [63] }\end{array}$ & $\begin{array}{l}\text { Workplace antistigma initiatives: A scoping } \\
\text { study }\end{array}$ & scoping review & 22 & $\begin{array}{l}\text { Australia, Canada, UK, } \\
\text { USA }\end{array}$ \\
\hline Mascayano et al. (2016) [63] & $\begin{array}{l}\text { Stigma toward mental illness in Latin America } \\
\text { and the Caribbean: A systematic review }\end{array}$ & integrative review & 26 & $\begin{array}{l}\text { Mexico, Brazil, Argentina, } \\
\text { Jamaica, Colombia, Peru, } \\
\text { Chile }\end{array}$ \\
\hline $\begin{array}{l}\text { McPherson \& Armstrong } \\
\text { (2012) [64] }\end{array}$ & $\begin{array}{l}\text { General practitioner management of } \\
\text { depression: a systematic review }\end{array}$ & $\begin{array}{l}\text { qualitative systematic } \\
\text { review with } \\
\text { assessment of } \\
\text { methodological } \\
\text { quality }\end{array}$ & 13 & UK, Sweden, Canada \\
\hline Mehta et al. (2015) [65] & $\begin{array}{l}\text { Evidence for effective interventions to reduce } \\
\text { mental health-related stigma and } \\
\text { discrimination in the medium and long term: } \\
\text { Systematic review }\end{array}$ & $\begin{array}{l}\text { quantitative } \\
\text { systematic review } \\
\text { with assessment of } \\
\text { methodological } \\
\text { quality }\end{array}$ & 80 & $\begin{array}{l}\text { USA, Canada, Australia, } \\
\text { UK, New Zealand, Italy, } \\
\text { Japan, Norway, Finland, } \\
\text { Greece, Hong Kong, } \\
\text { Germany, Turkey, Serbia, } \\
\text { China, India }\end{array}$ \\
\hline $\begin{array}{l}\text { Mestdagh \& Hansen (2014) } \\
\text { [66] }\end{array}$ & $\begin{array}{l}\text { Stigma in patients with schizophrenia } \\
\text { receiving community mental health care: a } \\
\text { review of qualitative studies }\end{array}$ & meta-ethnography & 18 & $\begin{array}{l}\text { UK, USA, Europe, } \\
\text { Australia, Turkey, Brazil, } \\
\text { Malaysia }\end{array}$ \\
\hline Milton \& Mullan (2014) [67] & Diagnosis telling in people with psychosis & integrative review & 14 & Not available \\
\hline Mittal et al. (2012) [68] & $\begin{array}{l}\text { Empirical studies of self-stigma reduction } \\
\text { strategies: A critical review of the literature }\end{array}$ & $\begin{array}{l}\text { quantitative } \\
\text { systematic review } \\
\text { with no assessment } \\
\text { of methodological } \\
\text { quality }\end{array}$ & 14 & $\begin{array}{l}\text { USA, Canada, Australia, } \\
\text { UK, Finland, China }\end{array}$ \\
\hline Mueller et al. (2016) [69] & $\begin{array}{l}\text { Communications to children about mental } \\
\text { illness and their role in stigma development: } \\
\text { An integrative review }\end{array}$ & integrative review & 15 & $\begin{array}{l}\text { UK, Finland, Ireland, } \\
\text { Canada, USA, Australia, } \\
\text { New Zealand, Spain }\end{array}$ \\
\hline $\begin{array}{l}\text { Parcesepe \& Cabassa et al. } \\
\text { (2013) [70] }\end{array}$ & $\begin{array}{l}\text { Public stigma of mental illness in the United } \\
\text { States: A systematic literature review }\end{array}$ & $\begin{array}{l}\text { quantitative } \\
\text { systematic review } \\
\text { with no assessment } \\
\text { of methodological } \\
\text { quality }\end{array}$ & 36 & USA \\
\hline Putman (2008) [71] & Mental illness: Diagnostic title or derogatory & integrative review & 31 & Not available \\
\hline
\end{tabular}
term? (Attitudes towards mental illness) Developing a learning resource for use within a clinical call centre. A systematic literature review on attitudes towards mental illness 
Table 1 Overview of 98 systematic reviews on stigma and HIV/AIDS, mental illness, and physical disability (Continued)

\begin{tabular}{|c|c|c|c|c|}
\hline Author(s), publication year & Title of paper & $\begin{array}{l}\text { Type(s) of systematic } \\
\text { review }\end{array}$ & $\begin{array}{l}\text { Number of } \\
\text { primary studies } \\
\text { included in the } \\
\text { review }\end{array}$ & $\begin{array}{l}\text { Geographic location(s) } \\
\text { of the primary studies }\end{array}$ \\
\hline Read et al. (2006) [72] & $\begin{array}{l}\text { Prejudice and schizophrenia: a review of the } \\
\text { 'mental illness is an illness like any other' } \\
\text { approach }\end{array}$ & $\begin{array}{l}\text { quantitative } \\
\text { systematic review } \\
\text { with no assessment } \\
\text { of methodological } \\
\text { quality }\end{array}$ & $\mathrm{n} / \mathrm{a}$ & $\begin{array}{l}\text { USA, England, Australia, } \\
\text { Japan, South Africa, } \\
\text { Ireland, India, Turkey, } \\
\text { Malaysia, China, Italy, } \\
\text { Ethiopia, Greece, Russia, } \\
\text { and Mongolia }\end{array}$ \\
\hline Schnyder et al. (2017) [73] & $\begin{array}{l}\text { Association between mental health-related } \\
\text { stigma and active help-seeking: Systematic } \\
\text { review and meta-analysis }\end{array}$ & $\begin{array}{l}\text { quantitative } \\
\text { systematic review } \\
\text { with no assessment } \\
\text { of methodological } \\
\text { quality and meta- } \\
\text { analysis }\end{array}$ & 27 & Not available \\
\hline Schomerus et al. (2012) [74] & $\begin{array}{l}\text { Evolution of public attitudes about mental } \\
\text { illness: A systematic review and meta-analysis }\end{array}$ & meta-analysis & 33 & Not available \\
\hline Seroalo et al. (2014) [2] & $\begin{array}{l}\text { A critical synthesis of interventions to reduce } \\
\text { stigma attached to mental illness }\end{array}$ & critical synthesis & 17 & $\begin{array}{l}\text { Hong Kong, USA, Russia, } \\
\text { Britain, Germany, } \\
\text { Sweden, Turkey }\end{array}$ \\
\hline Sharac et al. (2010) [3] & $\begin{array}{l}\text { The economic impact of mental health stigma } \\
\text { and discrimination: A systematic review }\end{array}$ & integrative review & 30 & $\begin{array}{l}\text { USA, Germany, Hong } \\
\text { Kong, Canada, New } \\
\text { Zealand, Israel, UK }\end{array}$ \\
\hline Sharp et al. (2015) [75] & $\begin{array}{l}\text { Stigma as a barrier to seeking health care } \\
\text { among military personnel with mental health } \\
\text { problems }\end{array}$ & $\begin{array}{l}\text { quantitative } \\
\text { systematic review } \\
\text { with assessment of } \\
\text { methodological } \\
\text { quality }\end{array}$ & 20 & USA, UK, Canada \\
\hline Stubbs (2014) [76] & $\begin{array}{l}\text { Reducing mental illness stigma in health care } \\
\text { students and professionals: A review of the } \\
\text { literature }\end{array}$ & $\begin{array}{l}\text { quantitative } \\
\text { systematic review } \\
\text { with no assessment } \\
\text { of methodological } \\
\text { quality }\end{array}$ & 18 & Not available \\
\hline Thornicroft et al. (2016) [77] & $\begin{array}{l}\text { Evidence for effective interventions to reduce } \\
\text { mental-health-related stigma and } \\
\text { discrimination }\end{array}$ & $\begin{array}{l}\text { quantitative } \\
\text { systematic review } \\
\text { with no assessment } \\
\text { of methodological } \\
\text { quality }\end{array}$ & 89 & Not available \\
\hline Tsang et al. (2016) [78] & $\begin{array}{l}\text { Therapeutic intervention for internalized } \\
\text { stigma of severe mental illness: A systematic } \\
\text { review and meta-analysis }\end{array}$ & $\begin{array}{l}\text { quantitative } \\
\text { systematic review } \\
\text { with assessment of } \\
\text { methodological } \\
\text { quality and meta- } \\
\text { analysis }\end{array}$ & 14 & $\begin{array}{l}\text { US, Canada, Turkey, } \\
\text { Hong Kong, Israel, } \\
\text { Switzerland, Austria, } \\
\text { Netherlands, Japan }\end{array}$ \\
\hline $\begin{array}{l}\text { Tzouvara \& Nyblade. (2016) } \\
\text { [79] }\end{array}$ & $\begin{array}{l}\text { Systematic review of the prevalence of mental } \\
\text { illness stigma within the Greek culture }\end{array}$ & $\begin{array}{l}\text { quantitative } \\
\text { systematic review } \\
\text { with no assessment } \\
\text { of methodological } \\
\text { quality }\end{array}$ & 18 & $\begin{array}{l}\text { Greece, United } \\
\text { Kingdom, Australia, } \\
\text { Cyprus }\end{array}$ \\
\hline Wittkowski et al. (2014) [80] & Exploring psychosis and bipolar disorder in & meta-ethnography & 13 & USA, Canada, UK, Japan \\
\hline
\end{tabular}
women: A critical review of the qualitative literature

Wood et al. (2016) [81] Psychosocial interventions for internalised stigma in people with a schizophreniaspectrum diagnosis: A systematic narrative synthesis and meta-analysis

Xu et al. (2017) [82] C Challenging mental health related stigma in China: Systematic review and meta-analysis. I. Interventions among the general public

quantitative

systematic review with assessment of methodological quality and metaanalysis

USA, England, Australia, and Mongolia

(n)


Table 1 Overview of 98 systematic reviews on stigma and HIV/AIDS, mental illness, and physical disability (Continued)

\begin{tabular}{|c|c|c|c|c|}
\hline Author(s), publication year & Title of paper & $\begin{array}{l}\text { Type(s) of systematic } \\
\text { review }\end{array}$ & $\begin{array}{l}\text { Number of } \\
\text { primary studies } \\
\text { included in the } \\
\text { review }\end{array}$ & $\begin{array}{l}\text { Geographic location(s) } \\
\text { of the primary studies }\end{array}$ \\
\hline Yamaguchi et al. (2011) [83] & $\begin{array}{l}\text { Strategies and future attempts to reduce } \\
\text { stigmatization and increase awareness of } \\
\text { mental health problems among young } \\
\text { people: A narrative review of educational } \\
\text { interventions }\end{array}$ & $\begin{array}{l}\text { quantitative } \\
\text { systematic review } \\
\text { with no assessment } \\
\text { of methodological } \\
\text { quality }\end{array}$ & 40 & Not available \\
\hline Yamaguchi et al. (2013) [84] & $\begin{array}{l}\text { Effects of short-term interventions to reduce } \\
\text { mental health-related stigma in university or } \\
\text { college students: A systematic review }\end{array}$ & $\begin{array}{l}\text { quantitative } \\
\text { systematic review } \\
\text { with assessment of } \\
\text { methodological } \\
\text { quality }\end{array}$ & 35 & $\begin{array}{l}\text { USA, Taiwan, UK, Japan, } \\
\text { Turkey, Germany }\end{array}$ \\
\hline Yamaguchi et al. (2017) [85] & $\begin{array}{l}\text { Associations between renaming schizophrenia } \\
\text { and stigma-related outcomes: A systematic } \\
\text { review }\end{array}$ & $\begin{array}{l}\text { quantitative } \\
\text { systematic review } \\
\text { with assessment of } \\
\text { methodological } \\
\text { quality }\end{array}$ & 23 & $\begin{array}{l}\text { Japan, South Korea, UK, } \\
\text { China, Canada, Ireland, } \\
\text { Turkey }\end{array}$ \\
\hline Yang et al. (2014) [86] & $\begin{array}{l}\text { Recent advances in cross-cultural measure- } \\
\text { ment in psychiatric epidemiology: Utilizing } \\
\text { 'what matters most' to identify culture-specific } \\
\text { aspects of stigma }\end{array}$ & integrative review & 196 & $\begin{array}{l}\text { USA, Asia/Pacific Islands, } \\
\text { Middle East, Africa, } \\
\text { Western Europe }\end{array}$ \\
\hline \multicolumn{5}{|l|}{ HIV/AIDS } \\
\hline Bharat (2011) [17] & $\begin{array}{l}\text { A systematic review of HIV/AIDS-related } \\
\text { stigma and discrimination in India: Current } \\
\text { understanding and future needs }\end{array}$ & integrative review & 30 & India \\
\hline Campbell et al. (2011) [87] & $\begin{array}{l}\text { Creating social spaces to tackle AIDS-related } \\
\text { stigma: Reviewing the role of church groups } \\
\text { in sub-Saharan Africa }\end{array}$ & integrative review & 36 & $\begin{array}{l}\text { Kenya, South Africa, } \\
\text { Uganda, Nigeria, } \\
\text { Tanzania, Burkina Faso, } \\
\text { East Africa, Ghana, } \\
\text { Ethiopia, Malawi, } \\
\text { Namibia, Zambia, } \\
\text { Zimbabwe, } \\
\text { Mozambique, Botswana, } \\
\text { Senegal, DR Congo, }\end{array}$ \\
\hline Columbini et al. (2014) [88] & $\begin{array}{l}\text { Factors affecting adherence to short-course } \\
\text { ARV prophylaxis for preventing mother-to- } \\
\text { child transmission of HIV in sub-Saharan Africa: } \\
\text { A review and lessons for future elimination }\end{array}$ & $\begin{array}{l}\text { quantitative } \\
\text { systematic review } \\
\text { with no assessment } \\
\text { of methodological } \\
\text { quality }\end{array}$ & 14 & $\begin{array}{l}\text { South Africa, Ethiopia, } \\
\text { Uganda, Zambia, } \\
\text { Zimbabwe, Cameroon, } \\
\text { Ivory Coast, Rwanda, } \\
\text { Kenya }\end{array}$ \\
\hline Dao et al. (2013) [89] & $\begin{array}{l}\text { Social science research on HIV in Vietnam: A } \\
\text { critical review and future directions }\end{array}$ & $\begin{array}{l}\text { qualitative systematic } \\
\text { review with no } \\
\text { assessment of } \\
\text { methodological } \\
\text { quality and mixed } \\
\text { methods review }\end{array}$ & 64 & Vietnam \\
\hline $\begin{array}{l}\text { Darlington \& Hutson (2017) } \\
\text { [26] }\end{array}$ & $\begin{array}{l}\text { Understanding HIV-related stigma among } \\
\text { women in the southern United States: A } \\
\text { literature review }\end{array}$ & integrative review & 27 & United States \\
\hline $\begin{array}{l}\text { Earnshaw \& Chaudoir } \\
\text { (2009) [19] }\end{array}$ & $\begin{array}{l}\text { From conceptualizing to measuring HIV } \\
\text { stigma: A review of HIV stigma mechanism } \\
\text { measures }\end{array}$ & $\begin{array}{l}\text { quantitative } \\
\text { systematic review } \\
\text { with no assessment } \\
\text { of methodological } \\
\text { quality }\end{array}$ & 23 & $\begin{array}{l}\text { USA, South Africa, Kenya, } \\
\text { Lesotho, Malawi, } \\
\text { Swaziland, Tanzania, } \\
\text { Thailand, India, China }\end{array}$ \\
\hline $\begin{array}{l}\text { Florom-Smith et al. (2012) } \\
\text { [90] }\end{array}$ & Exploring the concept of HIV-related stigma & integrative review & 21 & USA \\
\hline Gesesew et al. (2017) [91] & $\begin{array}{l}\text { Significant association between perceived HIV } \\
\text { related stigma and late presentation for HIV/ } \\
\text { AIDS care in low and middle-income countries: } \\
\text { A systematic review and meta-analysis }\end{array}$ & $\begin{array}{l}\text { quantitative } \\
\text { systematic review } \\
\text { with assessment of } \\
\text { methodological } \\
\text { quality and meta- } \\
\text { analysis }\end{array}$ & 10 & $\begin{array}{l}\text { Ethiopia, Venezuala, } \\
\text { Mexico, Brazil, } \\
\text { Zimbabwe, Kenya }\end{array}$ \\
\hline
\end{tabular}


Table 1 Overview of 98 systematic reviews on stigma and HIV/AIDS, mental illness, and physical disability (Continued)

\begin{tabular}{|c|c|c|c|c|}
\hline Author(s), publication year & Title of paper & $\begin{array}{l}\text { Type(s) of systematic } \\
\text { review }\end{array}$ & $\begin{array}{l}\text { Number of } \\
\text { primary studies } \\
\text { included in the } \\
\text { review }\end{array}$ & $\begin{array}{l}\text { Geographic location(s) } \\
\text { of the primary studies }\end{array}$ \\
\hline Ho \& Holloway. (2015) [92] & $\begin{array}{l}\text { The impact of HIV-related stigma on the lives } \\
\text { of HIV-positive women: An integrated } \\
\text { literature review }\end{array}$ & integrative review & 26 & $\begin{array}{l}\text { USA, South Africa, } \\
\text { Canada, UK, Tanzania, } \\
\text { Peru, Vietnam, Kenya, } \\
\text { New Zealand, Thailand, } \\
\text { India }\end{array}$ \\
\hline Katz et al. (2013) [93] & $\begin{array}{l}\text { Impact of HIV-related stigma on treatment } \\
\text { adherence: Systematic review and meta- } \\
\text { synthesis }\end{array}$ & integrative review & 75 & Not available \\
\hline Kerrigan et al. (2015) [16] & $\begin{array}{l}\text { A community empowerment approach to the } \\
\text { HIV response among sex workers: } \\
\text { Effectiveness, challenges, and considerations } \\
\text { for implementation and scale-up }\end{array}$ & meta-analysis. & 22 & $\begin{array}{l}\text { India, Brazil, Dominican } \\
\text { Republic }\end{array}$ \\
\hline Logie \& Gadalla (2009) [94] & $\begin{array}{l}\text { Meta-analysis of health and demographic } \\
\text { correlates of stigma towards people living } \\
\text { with HIV }\end{array}$ & meta-analysis & 24 & North America \\
\hline Lorenc et al. 2011 [95] & $\begin{array}{l}\text { HIV testing among men who have sex with } \\
\text { men (MSM): systematic review of qualitative } \\
\text { evidence }\end{array}$ & $\begin{array}{l}\text { qualitative systematic } \\
\text { review with } \\
\text { assessment of } \\
\text { methodological } \\
\text { quality }\end{array}$ & 17 & USA, UK, Canada \\
\hline Loutfy et al. (2015) [21] & $\begin{array}{l}\text { Systematic review of stigma reducing } \\
\text { interventions for African/Black diasporic } \\
\text { women }\end{array}$ & $\begin{array}{l}\text { quantitative } \\
\text { systematic review } \\
\text { with assessment of } \\
\text { methodological } \\
\text { quality }\end{array}$ & 5 & USA \\
\hline Lowther et al. (2014) [4] & $\begin{array}{l}\text { Experience of persistent psychological } \\
\text { symptoms and perceived stigma among } \\
\text { people with HIV on antiretroviral therapy } \\
\text { (ART): A systematic review }\end{array}$ & $\begin{array}{l}\text { quantitative } \\
\text { systematic review } \\
\text { with assessment of } \\
\text { methodological } \\
\text { quality }\end{array}$ & 66 & $\begin{array}{l}\text { USA, France, Italy, } \\
\text { Australia, Canada, Spain, } \\
\text { Netherlands, Portugal } \\
\text { and Sweden, South } \\
\text { Africa, India, Nigeria, } \\
\text { Botswana, Brazil, } \\
\text { Thailand, Uganda, } \\
\text { Cameroon, China, } \\
\text { Gambia, Jamaica, } \\
\text { Rwanda, Senegal, } \\
\text { Vietnam, Zambia }\end{array}$ \\
\hline Mahajan et al. (2008) [96] & $\begin{array}{l}\text { Stigma in the HIV/AIDS epidemic: a review of } \\
\text { the literature and recommendations for the } \\
\text { way forward }\end{array}$ & integrative review & 390 & $\begin{array}{l}\text { North America, Western } \\
\text { Europe }\end{array}$ \\
\hline Mak et al. (2017) [97] & $\begin{array}{l}\text { Meta-analysis and systematic review of studies } \\
\text { on the effectiveness of HIV stigma reduction } \\
\text { programs }\end{array}$ & $\begin{array}{l}\text { qualitative systematic } \\
\text { review with } \\
\text { assessment of } \\
\text { methodological } \\
\text { quality and meta- } \\
\text { analysis }\end{array}$ & $m-42 . q s-35$ & USA, Africa, Europe, Asia \\
\hline Maulsby et al. (2014) [27] & $\begin{array}{l}\text { HIV among Black Men who have Sex with } \\
\text { Men (MSM) in the United States: A review of } \\
\text { the literature }\end{array}$ & integrative review & 39 & USA \\
\hline McAteer et al. (2017) [98] & $\begin{array}{l}\text { A systematic review of measures of HIV/AIDS } \\
\text { stigma in paediatric HIV-infected and HIV- } \\
\text { affected populations }\end{array}$ & $\begin{array}{l}\text { qualitative systematic } \\
\text { review with } \\
\text { assessment of } \\
\text { methodological } \\
\text { quality }\end{array}$ & 22 & $\begin{array}{l}\text { United States, Africa, } \\
\text { Asia, Sweden }\end{array}$ \\
\hline Mills et al. (2006) [99] & $\begin{array}{l}\text { Barriers to participation in HIV drug trials: A } \\
\text { systematic review }\end{array}$ & integrative review & 14 & $\begin{array}{l}\text { USA, EU, Australia, } \\
\text { France, UK }\end{array}$ \\
\hline Monjok et al. (2009) [100] & HIV/AIDS - related stigma and discrimination & integrative review & 8 & Nigeria \\
\hline
\end{tabular}


Table 1 Overview of 98 systematic reviews on stigma and HIV/AIDS, mental illness, and physical disability (Continued)

\begin{tabular}{lll}
\hline Author(s), publication year & Title of paper & $\begin{array}{l}\text { Type(s) of systematic } \\
\text { review }\end{array}$ \\
\hline Monteiro et al. (2013) [22] & $\begin{array}{l}\text { The interaction between axes of inequality in } \\
\text { studies on discrimination, stigma and HIV/ } \\
\text { AlDS: Contributions to the recent international } \\
\text { literature }\end{array}$
\end{tabular}

Monteiro et al. (2012) [101] Discrimination, stigma, and AIDS: A review of academic literature produced in Brazil (20052010)

Paudel \& Baral (2015) [102] Women living with HIV/AIDS (WLHA), battling stigma, discrimination and denial and the role of support groups as a coping strategy: a review of literature

Prost et al. (2008) [103]

Social, behavioural, and intervention research among people of sub-Saharan African origin living with HIV in the UK and Europe:

Literature review and recommendations for intervention

Roger et al. (2013) [104]

Social aspects of HIV/AIDS and aging: A thematic review

Sandelowski et al. (2009)

[23]

Gender, race/ethnicity, and social class in research reports on stigma in HIV-positive women

Sengupta et al. (2011) [105] HIV interventions to reduce HIV/AIDS stigma: A systematic review

Smith et al. (2008) [106]

A meta-analysis of disclosure of one's HIVpositive status, stigma and social support

Stangl et al. (2013) [15]

A systematic review of interventions to reduce HIV-related stigma and discrimination from 2002 to 2013: How far have we come?
Number of

primary studies

included in the

review

42

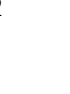

meta-ethnography and mixed methods review

meta-ethnography

integrative review

138

163

\section{7}

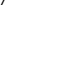

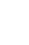

.

\section{Geographic location(s) \\ of the primary studies}

Jamaica, USA, Nepal,

Jamaica, USA, Nepal,

Uganda, India, Puerto

Rico, England, Mexico,

Guatemala, Trinidad and

Tobago, Bangladesh,

Kenya, South Korea,

Dominican Republic,

Malawi, China, Tanzania,

South Africa, Canada

Brazil

Canada, India, Uganda, Australia, Tanzania, United States of America, Thailand

Europe

integrative review

62

Canada

meta-study

USA

quantitative

systematic review with assessment of methodological quality

meta-analysis

integrative review

(n)

\begin{abstract}
Sweeney \& Vanable (2016) The association of HIV-related stigma to HIV [107]

medication adherence: A systematic review and synthesis of the literature
\end{abstract}

quantitative systematic review with no assessment of methodological quality

integrative review stigma derived from a review of the HIVaffected couples literature

Talley \& Bettencourt (2010) [108]

Turan \& Nyblade (2013) [79] of global PMTCT and maternal health goals: A review of the evidence

Weihs \& Meyer-Weitz (2016) Barriers to workplace HIV testing in South

\section{integrative review}

integrative review

Integrative review
Not available Netherlands
North America, Europe, Asia, Africa

USA, UK, South Africa, India.

Saudi Arabia, South Africa, Zambia, China, India, Uganda, Chile, Ethiopia, Australia, China, Ghana, Nigeria, Malawi, Hong Kong, Angola, Cameroon, Ivory Coast, Equatorial Guinea, Kenya, Haiti, Peru, Thailand, USA, Swaziland, Tanzania, Vietnam Canada

USA, South Africa, Kenya, India, Thailand, China, Tanzania, Hong Kong, Democratic Republic of Congo, Ethiopia, Zambia, Nigeria, France, The

Thailand, India, USA 
Table 1 Overview of 98 systematic reviews on stigma and HIV/AIDS, mental illness, and physical disability (Continued)

\begin{tabular}{|c|c|c|c|c|}
\hline Author(s), publication year & Title of paper & $\begin{array}{l}\text { Type(s) of systematic } \\
\text { review }\end{array}$ & $\begin{array}{l}\text { Number of } \\
\text { primary studies } \\
\text { included in the } \\
\text { review }\end{array}$ & $\begin{array}{l}\text { Geographic location(s) } \\
\text { of the primary studies }\end{array}$ \\
\hline$[109]$ & Africa: A systematic review of the literature & & & \\
\hline \multicolumn{5}{|l|}{ Physical disability } \\
\hline Boyles et al. (2008) [14] & $\begin{array}{l}\text { Representations of disability in nursing and } \\
\text { healthcare literature: an integrative review }\end{array}$ & integrative review & 65 & Not available \\
\hline Wilson et al. (2013) [110] & $\begin{array}{l}\text { Attitudes towards individuals with disabilities } \\
\text { as measured by the Implicit Association Test: } \\
\text { A literature review }\end{array}$ & $\begin{array}{l}\text { quantitative } \\
\text { systematic review } \\
\text { with assessment of } \\
\text { methodological } \\
\text { quality }\end{array}$ & 17 & $\begin{array}{l}\text { USA, China, Italy, UK, } \\
\text { Germany, France }\end{array}$ \\
\hline Zeldenryk et al. (2011) [111] & $\begin{array}{l}\text { The emerging story of disability associated } \\
\text { with lymphatic filariasis: A critical review }\end{array}$ & $\begin{array}{l}\text { qualitative systematic } \\
\text { review with } \\
\text { assessment of } \\
\text { methodological } \\
\text { quality }\end{array}$ & 16 & $\begin{array}{l}\text { Ghana, India, Haiti, Sri } \\
\text { Lanka, Dominican } \\
\text { Republic }\end{array}$ \\
\hline \multicolumn{5}{|l|}{ Combination papers } \\
\hline Van Bakel (2007) [13] & $\begin{array}{l}\text { Measuring health-related stigma- a literature } \\
\text { review }\end{array}$ & $\begin{array}{l}\text { quantitative } \\
\text { systematic review } \\
\text { with no assessment } \\
\text { of methodological } \\
\text { quality }\end{array}$ & 63 & $\begin{array}{l}\text { Most instruments } \\
\text { developed for use in the } \\
\text { USA }\end{array}$ \\
\hline
\end{tabular}

stigma, experienced stigma), and just 3\% focused on institutional/structural stigma (i.e. treatment stigma).

Interventions to manage, reduce, and prevent stigma were included in $36 \%(n=35)$ of the reviews. Among these, interventions for mental illness stigma were the most common $(74 \%, n=26)$, followed by interventions for HIV/AIDS stigma $(23 \%, n=8)$, and a smaller number of interventions for physical disability stigma $(3 \%, n=1)$ (See Table 4 for a complete list of interventions and their characteristics).

Behavioural interventions such as psychoeducation, informational approaches, and/or social contact were most commonly reported in the reviews of interventions (94\%, $n=33$ ). In comparison, only one review of HIV/AIDS stigma described structural interventions. In this latter review, a structural intervention (universal precaution supplies to healthcare workers) was combined with a behavioural intervention focusing on information and skill-building to combat HIV/AIDS stigma [15]. Additionally, only 3 reviews described community-based interventions, which included a community-empowerment approach to respond to HIV/AIDS among sex workers [16], community mobilization and involvement to address HIV/AIDS stigma in India [17], and advocacy-based approaches for mental illness stigma in Canada [18].

\section{Intersectionality}

Our text search yielded 13 reviews (17\%) whose authors had used an intersectional lens to analyze primary research studies. The majority of these reviews were found in the work on HIV/AIDS stigma $(92 \%, n=12)$; just one review of mental illness stigma (8\%) used intersectionality. While all 13 of these reviews mentioned intersectionality when describing how a mental illness or HIV/AIDS diagnosis intersected with culture, power and/or other differences to reinforce social conditions for stigmatization [19, 20], only three of these 13 reviews (23\%) [21-23] provided a definition for intersectionality. Loutfy et al. [21] and Monteiro et al.'s [22] used Crenshaw's [6, 7] concept of intersectionality in discussing how health inequities are impacted by categories of difference like HIV status, race, gender, and sexuality. Both reviews used this concept of intersectionality to analyze the primary studies and highlighted its usefulness in improving knowledge about how processes of marginalization overlap and are impacted by HIV/AIDS stigma [21, 22]. Sandelowski's [23] review used a more recent concept of intersectionality, which characterized it as a research paradigm and methodological intervention [24, 25]. For instance, Sandelowski [23] explained that intersectionality acknowledges intra-category diversity and can help to investigate relationships between and among dynamic categories of difference like gender in health research. However, Sandelowski [23] found that non-intersectional, unitary analytical approaches that isolated overlapping categories of difference like race, gender, and HIV stigma from one another were overwhelmingly used in the primary research studies.

\section{Intersectional stigma and interventions}

Five of the reviews that included intersectionality used it as a framework to discuss the occurrence of intersectional 
Table 2 Types of systematic reviews, definitions, and references

\begin{tabular}{ll}
\hline Type of systematic review & Definition and reference(s) \\
\hline Critical synthesis & A review which aims to demonstrate that the writer has extensively researched the literature and critically \\
& evaluated its quality. This review technique incorporates analysis and conceptual innovation [112]. \\
Integrative review & $\begin{array}{l}\text { A technique that integrates review, critique, and synthesis of representative literature on a topic to create new } \\
\text { frameworks and perspectives on the topic [113]. It also includes experimental and non-experimental research } \\
\text { studies and combines theoretical and empirical data to gain a more comprehensive understandings of a } \\
\text { phenomenon [114] . }\end{array}$ \\
& $\begin{array}{l}\text { A review technique that "systematically combines the results of quantitative studies to provide a more precise } \\
\text { effect of the results" [112]. }\end{array}$ \\
Meta-analysis & $\begin{array}{l}\text { An interpretive and inductive approach that combines and sometimes compare the findings of ethnographic } \\
\text { research or qualitative research to provide a higher level of analysis, generate new research questions, and reduce } \\
\text { duplicate research [115, 116]. }\end{array}$ \\
& $\begin{array}{l}\text { A research approach that involves the analysis of theory, methods, and findings of qualitative research and } \\
\text { synthesizes these insights into new ways of thinking about some phenomena [117]. }\end{array}$ \\
Meta-study & A review technique that combines qualitative and quantitative approaches [112]. \\
Mixed methods review & A research approach that provides a "preliminary assessment of the potential size and scope" of the research on \\
Scoping review & $\begin{array}{l}\text { a particular subject. The aim of this review is to identify the nature and size of research [112]. } \\
\text { Engages in a systematized search, appraisal, and synthesis of research evidence which adheres to a set of } \\
\text { guidelines [112]. Some qualitative and quantitative reviews include an assessment of methodological quality, while } \\
\text { others may not. }\end{array}$ \\
\hline
\end{tabular}

stigma in the primary research studies. This process was defined in Loutfy et al.'s [21] review as the "multiple, simultaneous and dynamic interchanges among categories of social difference as it interlinks with power and privilege, and systemic oppression and its operation at the micro, mesa, and macro levels" (p. 2). The HIV/AIDS reviews tended to focus on stigma amongst groups that have been socially and historically marginalized such as Black women [21, 26], Black men who have sex with men [27], and sex workers [16]. In these reviews, focusing on intersectional stigma allowed other forms of social inequality experienced by people living with HIV/AIDS like racism, sexism, and homophobia to be included and highlighted the existence of overlapping forms of oppression and marginalization.

Although interventions for stigma were described in $36 \%$ of the reviews, none of the authors indicated whether or how intersectional interventions were used in the primary studies. However, three of the 35 reviews (8\%) highlighted the lack of intersectional interventions designed to address intersectional stigma in the primary studies, and advocated for their use in primary research studies [15, 21]. Loutfy et al.'s [21] review found there was an absence of stigma-reducing interventions that addressed co-occurring stigmas experienced by Black women who are HIV positive, and that most focused on interpersonal and intrapersonal stigma. Kerrigan et al.'s [16] review of community-empowerment interventions to counter HIV stigma amongst mainly female sex workers found that one of the greatest structural barriers to the implementation and scale-up of these interventions was the presence of intersectional stigma.

\section{Discussion}

To our knowledge, this is the first review to provide a cross-analysis of systematic reviews of HIV/AIDS, mental illness, and physical disability stigma. It is also the first review of reviews to examine whether and how intersectionality has been used as an analytic approach on stigma.

The nearly complete lack of reviews that examined stigma across these three health conditions may be partly attributed to trends in stigma research [28], since systematic review topics are constrained by primary studies on a topic. Researchers conducting effectiveness studies of interventions concentrate on specific target

Table 3 Types and definitions of stigma discussed in 98 systematic reviews

\begin{tabular}{|c|c|c|c|}
\hline Stigma Type & Definition & Number & $\%^{a}$ \\
\hline Intrapersonal Stigma & $\begin{array}{l}\text { When an individual internalizes publicly held negative beliefs about a health condition, and applies them } \\
\text { to her or himself }\end{array}$ & 77 & 78.5 \\
\hline Interpersonal Stigma & $\begin{array}{l}\text { The process in which members of the general public direct stigma towards individuals with a specific } \\
\text { health condition }\end{array}$ & 47 & 48 \\
\hline $\begin{array}{l}\text { Institutional/Structural } \\
\text { Stigma }\end{array}$ & Practices initiated at the institutional level that work to disadvantage a stigmatized group or person & 3 & 3 \\
\hline
\end{tabular}


Table 4 Characteristics of the stigma interventions

\begin{tabular}{|c|c|c|c|c|}
\hline $\begin{array}{l}\text { Author(s), publication } \\
\text { year }\end{array}$ & $\begin{array}{l}\text { Health } \\
\text { Condition }\end{array}$ & $\begin{array}{l}\text { Institutional Interventions } \\
\text { (i.e. interventions } \\
\text { administered by hospitals, } \\
\text { healthcare institutions, etc.) }\end{array}$ & $\begin{array}{l}\text { Behavioural Interventions (i.e. social contact, } \\
\text { education-based, etc.) }\end{array}$ & $\begin{array}{l}\text { Community-based Interventions } \\
\text { (i.e. religious, ethno-racial, etc.) }\end{array}$ \\
\hline Ando et al. (2013) [34] & $\begin{array}{l}\text { Mental } \\
\text { Illness }\end{array}$ & & $\begin{array}{l}\text { Educational interventions. Contact-based } \\
\text { interventions }\end{array}$ & \\
\hline Bharat. (2011) [17] & HIV/AIDS & & & $\begin{array}{l}\text { Community mobilisation and } \\
\text { involvement. }\end{array}$ \\
\hline Clarke et al. (2014) [40] & $\begin{array}{l}\text { Mental } \\
\text { Illness }\end{array}$ & & Educational interventions & \\
\hline Clement et al. (2013) [42] & $\begin{array}{l}\text { Mental } \\
\text { Illness }\end{array}$ & & Mass media & \\
\hline Dalky (2012) [45] & $\begin{array}{l}\text { Mental } \\
\text { Illness }\end{array}$ & & $\begin{array}{l}\text { Educational interventions contact-based } \\
\text { interventions }\end{array}$ & \\
\hline $\begin{array}{l}\text { Darlington \& Hutson } \\
\text { (2017) [26] }\end{array}$ & HIV/AIDS & & Support groups, visual media interventions & \\
\hline Doley et al. (2017) [47] & $\begin{array}{l}\text { Mental } \\
\text { Illness }\end{array}$ & & $\begin{array}{l}\text { Educational interventions. Contact-based } \\
\text { interventions }\end{array}$ & \\
\hline Gerlinger et al. (2013) [52] & $\begin{array}{l}\text { Mental } \\
\text { Illness }\end{array}$ & & $\begin{array}{l}\text { Cognitive-behavioral therapy Educational } \\
\text { interventions }\end{array}$ & \\
\hline Griffiths et al. (2014) [53] & $\begin{array}{l}\text { Mental } \\
\text { Illness }\end{array}$ & & $\begin{array}{l}\text { Educational interventions Contact-based } \\
\text { interventions }\end{array}$ & \\
\hline Guruge et al. (2017) [18] & $\begin{array}{l}\text { Mental } \\
\text { Illness }\end{array}$ & & $\begin{array}{l}\text { Contact-based interventions Experiential } \\
\text { videos/photos Educational interventions }\end{array}$ & Advocacy based \\
\hline Hanisch et al. (2016) [54] & $\begin{array}{l}\text { Mental } \\
\text { Illness }\end{array}$ & & $\begin{array}{l}\text { Educational interventions Mental Health } \\
\text { First Aid (MHFA) training role play, Trauma } \\
\text { Risk Management (TRiM), and Crisis } \\
\text { Intervention Training (CIT) in first responders. }\end{array}$ & \\
\hline Haugen et al. (2017) [55] & $\begin{array}{l}\text { Mental } \\
\text { Illness }\end{array}$ & & Video & \\
\hline Hawke et al. (2013) [56] & $\begin{array}{l}\text { Mental } \\
\text { Illness }\end{array}$ & & $\begin{array}{l}\text { Educational interventions Contact-based } \\
\text { interventions }\end{array}$ & \\
\hline Jorm (2012) [58] & $\begin{array}{l}\text { Mental } \\
\text { Illness }\end{array}$ & & $\begin{array}{l}\text { Educational interventions Contact-based } \\
\text { interventions }\end{array}$ & \\
\hline Kerrigan et al. (2015) [16] & HIV/AIDS & & Educational interventions & Community-empowerment \\
\hline Loutfy et al. (2015) [21] & HIV/AIDS & & $\begin{array}{l}\text { Educational interventions Project ACCEPT } \\
\text { (Adolescents Coping, Connecting, } \\
\text { Empowering and Protecting Together) }\end{array}$ & \\
\hline Mak et al. (2017) [97] & HIV/AIDS & & Educational interventions & \\
\hline $\begin{array}{l}\text { Malachowski \& Kirsh. } \\
\text { (2013) [63] }\end{array}$ & $\begin{array}{l}\text { Mental } \\
\text { Illness }\end{array}$ & & Educational interventions & \\
\hline Mehta et al. (2015) [65] & $\begin{array}{l}\text { Mental } \\
\text { Illness }\end{array}$ & & Contact-based interventions & \\
\hline $\begin{array}{l}\text { Milton \& Mullan (2014) } \\
\text { [67] }\end{array}$ & $\begin{array}{l}\text { Mental } \\
\text { Illness }\end{array}$ & & Educational interventions (Psychoeducation) & \\
\hline Mittal et al. (2012) [68] & $\begin{array}{l}\text { Mental } \\
\text { Illness }\end{array}$ & & $\begin{array}{l}\text { Educational interventions (Psychoeducation } \\
\text { or psychoeducation combined with } \\
\text { cognitive restructuring, Psychoeducation } \\
\text { with complex multimodal interventions) }\end{array}$ & \\
\hline Mueller et al. (2016) [69] & & & Educational interventions for children & \\
\hline Parcesepe et al. (2013) [70] & $\begin{array}{l}\text { Mental } \\
\text { Illness }\end{array}$ & & Contact-based interventions & \\
\hline Prost et al. (2008) [103] & HIV/AIDS & & $\begin{array}{l}\text { Educational interventions Prevention } \\
\text { interventions. }\end{array}$ & \\
\hline Sengupta et al. (2011) & HIV/AIDS & & Educational interventions & \\
\hline
\end{tabular}


Table 4 Characteristics of the stigma interventions (Continued)

\begin{tabular}{|c|c|c|c|c|}
\hline $\begin{array}{l}\text { Author(s), publication } \\
\text { year }\end{array}$ & $\begin{array}{l}\text { Health } \\
\text { Condition }\end{array}$ & $\begin{array}{l}\text { Institutional Interventions } \\
\text { (i.e. interventions } \\
\text { administered by hospitals, } \\
\text { healthcare institutions, etc.) }\end{array}$ & $\begin{array}{l}\text { Behavioural Interventions (i.e. social contact, } \\
\text { education-based, etc.) }\end{array}$ & $\begin{array}{l}\text { Community-based Interventions } \\
\text { (i.e. religious, ethno-racial, etc.) }\end{array}$ \\
\hline Seroalo et al. (2014) [2] & $\begin{array}{l}\text { Mental } \\
\text { Illness }\end{array}$ & & $\begin{array}{l}\text { Educational interventions using theatrical } \\
\text { presentations with actors living with mental } \\
\text { illness. }\end{array}$ & \\
\hline Stangl et al. (2013) [15] & HIV/AIDS & $\begin{array}{l}\text { Universal precaution supplies } \\
\text { (first aid kits) }\end{array}$ & Information and skill building & \\
\hline Stubbs (2014) [76] & $\begin{array}{l}\text { Mental } \\
\text { Illness }\end{array}$ & & $\begin{array}{l}\text { Direct contact, indirect filmed contact, or } \\
\text { educational email. Role play }\end{array}$ & \\
\hline $\begin{array}{l}\text { Thornicroft et al. (2016) } \\
\text { [77] }\end{array}$ & $\begin{array}{l}\text { Mental } \\
\text { Illness }\end{array}$ & & $\begin{array}{l}\text { Education or information interventions, and } \\
\text { variants of social contact interventions }\end{array}$ & \\
\hline Tsang et al. (2016) [78] & $\begin{array}{l}\text { Mental } \\
\text { Illness }\end{array}$ & & Psychoeducation & \\
\hline Wilson et al. (2013) [110] & $\begin{array}{l}\text { Physical } \\
\text { Disability }\end{array}$ & & Implicit Association Test & \\
\hline Wood et al. (2016) [81] & $\begin{array}{l}\text { Mental } \\
\text { Illness }\end{array}$ & & $\begin{array}{l}\text { Psychosocial interventions: psychoeducation, } \\
\text { thought challenging, connecting with peers } \\
\text { and social skills training }\end{array}$ & \\
\hline Xu et al. (2017) [82] & $\begin{array}{l}\text { Mental } \\
\text { Illness }\end{array}$ & & $\begin{array}{l}\text { Education interventions either alone or in } \\
\text { combination with consumer contact, } \\
\text { including contact in person and via video. } \\
\text { Education strategies: lectures, role-plays, } \\
\text { videos, and educational materials }\end{array}$ & \\
\hline $\begin{array}{l}\text { Yamaguchi et al. (2013) } \\
\text { [84] }\end{array}$ & $\begin{array}{l}\text { Mental } \\
\text { Illness }\end{array}$ & & Social contact or video based social contact & \\
\hline $\begin{array}{l}\text { Yamaguchi et al. (2011) } \\
\text { [83] }\end{array}$ & $\begin{array}{l}\text { Mental } \\
\text { Illness }\end{array}$ & & $\begin{array}{l}\text { Contact-based interventions and education } \\
\text { interventions }\end{array}$ & \\
\hline
\end{tabular}

If the cell is empty, no descriptions were provided

groups and conditions, in part because the aim of effectiveness research is to determine whether a specific outcome can be attributed to a particular intervention. Thus, researchers doing effectiveness studies are more likely to focus on homogenous (e.g. populations with a primary diagnosis such as HIV/AIDS) rather than heterogeneous populations. There is also a predominance of disease-specific funding, which may preclude and/or discourage cross-analytical work. Funding calls that require stigma research on heterogeneous samples would foster this kind of work, enabling cross-analyses of stigma for these health conditions.

We noticed that in reviews of intervention studies, there is a dominant focus on behavioural rather than structural interventions for stigma, and that reviews typically focus on interpersonal and intrapersonal stigma rather than structural and institutional stigma. This gap may be due to a dearth of primary studies with a structural focus, reflecting a persistent person-centric orientation towards stigma reduction [29]. In addition to the need for more reviews that compare stigma and destigmatizing interventions across disease conditions, there is a need for reviews that compare behavioural and structural interventions or their combination. This would provide an important basis for comparing stigma reduction approaches with either (or both) orientations.

Our review indicates that research on stigma has begun to move in the direction of acknowledging the intersectionality of these experiences and grappling with how stigmatization overlaps with other forms of oppression. Although just one of the mental illness stigma reviews used intersectionality, it highlighted the potential value of intersectionality as an analytical framework, noting that it captured the interlocking effects of various kinds of oppression as it overlaps with mental illness stigma [30]. A small proportion of systematic reviews of HIV/AIDS stigma were categorized as using intersectionality frameworks. We think the integration of intersectionality within some HIV/AIDS reviews could be a result of the expansive body of diverse and cross-cultural, cross-racial, and cross-geographical research on HIV/AIDS stigma, which may have stimulated the application of analytical frameworks that acknowledge the convergence of multiple kinds of stigma and structural inequality.

The limited number of reviews addressing physical disability stigma is noteworthy. We do not know if this gap reflects a lack of primary research studies on this topic. Nevertheless, Boyles et al. [14] stated that 
historically, most research on disability has been designed, conducted, and managed by people who do not live with disabilities, which limits knowledge creation about these health conditions. This is in sharp contrast with research on HIV/AIDS, which has a long history of involving persons living with HIV/AIDS in many facets of research studies. If more opportunities were created to meaningfully include and facilitate leadership roles for people living with disabilities in health research, we might see an increase in both primary research and systematic reviews on this topic. Purposefully engaging those living with physical disability in studies on HIV/ AIDS and mental health would help to build the evidence-base on co-occurring stigma.

\section{Recommendations for future research}

This review of reviews indicates there is a need for more work that focuses on structural interventions to reduce stigma in both primary studies and systematic reviews. While several reviews highlighted the lack of structural interventions in their findings, we believe it is also imperative to identify concrete examples of these kinds of interventions when reviewers present recommendations for future work on this topic. At the organizational level, examples might include research that examines institutional interventions implemented to reduce stigma in healthcare settings like culturally-specific mental healthcare programs, clinical assessments that omit problematic or pathologizing questions about gender and sexuality, and anti-stigma training for healthcare professionals. At the state level, policies such as legalizing/decriminalizing homosexuality or enacting legislation that protects the rights of people living with disabilities and mental illness are examples of structural interventions that might be expected to have an impact on stigma.

To strengthen the reviews of stigma across these health conditions and in cross-comparative work we call for more explicit integration of intersectionality frameworks in the methodology of systematic reviews. It is not sufficient for reviews to use the language of intersectionality as an afterthought in the conclusion or discussion sections or to hint at co-occurring inequalities or people's multiple social identities without context, clear definitions, and critical reflections on how the term has evolved. Researchers need to work towards more accurate and meaningful inclusions of intersectional approaches, which use the concept to deepen analyses of stigma, particularly as it applies to understanding the presence of stigma from more than one health condition and from other co-occurring sources of stigma emanating from social identities like race, gender, and sexuality.

\section{Limitations}

We searched five reputable databases that publish medical and health research. However, we did not search databases that are exclusively in the social sciences and may have missed some pertinent reviews as a result. We did not use intersectionality as a search term. This may have led to the omission of some eligible reviews although that seems unlikely since our broader search terms likely captured any reviews that included intersectional approaches. Nevertheless, the patterns we observed are overwhelming and it seems unlikely that a different pattern would have emerged even if some additional reviews had been found.

We were cautious in our categorization of reviews as using or not using intersectional approaches, and reviews that included a discussion or analysis of other social categories like race or gender were not categorized as intersectional on that basis alone. Our conservative approach is consistent with a literature that warns against misappropriation of the concept, describing how it is sometimes used to gloss over identity politics in research or to treat categories like race and gender as independent variables rather than as reflections of social practices that are linked to larger processes of inequality $[9,30,31]$. We acknowledge that more conventional systematic review methods may not be congruent with reporting on this deeper and more nuanced approach to the intersectional analyses of stigma.

Our approach to identifying and selecting articles focused on reviews rather than primary studies. While it seems reasonable to conclude that the gaps we identified from systematic reviews mirror gaps among primary studies, we are not able to confirm this.

\section{Conclusions}

The nearly total lack of systematic reviews examining stigma across mental illness, HIV/AIDS, and physical disability indicates there are ripe opportunities for further primary research and systematic reviews that undertake a cross-comparative analyses among these health conditions. Approaches such as intersectionality that deepen our interrogation of intersecting stigma and that acknowledge and address larger processes of inequality and inequity that occur alongside health stigma are needed. Such approaches may inform intervention design as well as research methods; these are needed to avoid reproducing and exacerbating inequalities and inequities among population that experience marginalization due to their health condition(s).

\section{Abbreviations}

CINAHL: Cumulative Index to Nursing and Allied Health Literature; HIV/ AIDS: Human Immunodeficiency Virus Infection and Acquired Immune Deficiency Syndrome; PLWHA: People living with HIV/AIDS 


\section{Acknowledgments}

Thank you to Karine Fournier and Yeonjung Yoo for their assistance with this paper

\section{Funding}

FJB was supported by a University of Ottawa postdoctoral fellowship.

\section{Authors' contributions}

NE and FJB jointly developed the research questions and the research methods for the scoping review. FJB and NE co-developed the eligibility and extraction criteria and conducted an initial review of a sub-sample of articles to refine these criteria. FJB extracted, analyzed and summarized the research findings emanating from the systematic reviews that are presented in this manuscript. FJB interpreted the findings. FJB wrote an initial draft of the manuscript; NE provided edits and wrote portions of the results and discussion sections. Both authors contributed to final versions of the manuscript, and read and approved the final manuscript.

\section{Ethics approval and consent to participate}

Not applicable.

\section{Consent for publication}

Not applicable.

\section{Competing interests}

The authors declare that they have no competing interests.

\section{Publisher's Note}

Springer Nature remains neutral with regard to jurisdictional claims in published maps and institutional affiliations.

\section{Author details}

'I Am One, 58C Cypress Ridge, Union Hall, San Fernando, Trinidad and Tobago. ${ }^{2}$ School of Nursing, Faculty of Health Sciences, University of Ottawa, 1 Stewart Street, Room 205, Ottawa, ON K1N 7M9, Canada.

\section{Received: 30 March 2018 Accepted: 17 July 2018}

\section{Published online: 27 July 2018}

\section{References}

1. Parker R, Aggleton P. HIV and AIDS-related stigma and discrimination: a conceptual framework and implications for action. Soc Sci Med. 2003;57:1324.

2. Seroalo KB, Plessis ED, Koen MP, Koen V. A critical synthesis of interventions to reduce stigma attached to mental illness: original research. Health SA Gesondheid. 2014:19:1-10.

3. Sharac J, Mccrone P, Clement S, Thornicroft G. The economic impact of mental health stigma and discrimination: a systematic review. Epidemiol Psychiatr Sci. 2010;19:223-32.

4. Lowther K, Selman L, Harding R, Higginson IJ. Experience of persistent psychological symptoms and perceived stigma among people with HIV on antiretroviral therapy (ART): a systematic review. Int J Nurs Stud. 2014;51: $1171-89$.

5. Turner RJ, Noh S. Physical disability and depression: a longitudinal analysis. J Health Soc Behav. 1988;29:23-37.

6. Crenshaw K. Demarginalizing the intersection of race and sex: a black feminist critique of antidiscrimination doctrine, feminist theory and antiracist politics. U Chi Legal F. 1989;1989:139-67.

7. Crenshaw K. Mapping the margins: intersectionality, identity politics, and violence against women of color. Stanford Law Rev. 1991;43:1241.

8. Hill-Collins P. Black feminist thought: knowledge, consciousness, and the politics of empowerment. New York: Routledge; 2000.

9. Cole ER. Intersectionality and research in psychology. Am Psychol. 2009:64: $170-80$

10. Jackson-Best F. A narrative review of maternal depression research focusing on women of Caribbean descent in the diaspora and Caribbean women in the region. J Int Womens Stud. 2016;17(3):32-44.

11. Arksey H, O'Malley L. Scoping studies: towards a methodological framework Int J Soc Res Methodol. 2005;8:19-32.

12. Human Rights Code, RSO 1990, c H.19. http://canlii.ca/t/532d6. Accessed 1 July 2018
13. Van Brakel WH. Measuring health-related stigma--a literature review. Psychol Health Med. 2006:11:307-34.

14. Boyles CM, Bailey PH, Mossey S. Representations of disability in nursing and healthcare literature: an integrative review. J Adv Nurs. 2008;62:428-37.

15. Stangl AL, Lloyd JK, Brady LM, Holland CE, Baral S. A systematic review of interventions to reduce HIV-related stigma and discrimination from 2002 to 2013: how far have we come? J Int AIDS Soc. 2013;16(Suppl 2):18734.

16. Kerrigan D, Kennedy CE, Morgan-Thomas R, Reza-Paul S, Mwangi P, Win KT, et al. A community empowerment approach to the HIV response among sex workers: effectiveness, challenges, and considerations for implementation and scale-up. Lancet. 2015;385(9963):172-85.

17. Bharat S. A systematic review of HIV/AIDS-related stigma and discrimination in India: current understanding and future needs. J Soc Asp HIV. 2011:8:138-49.

18. Guruge $S$, Wang AZY, Jayasuriya-lllesinghe V, Sidani S. Knowing so much, yet knowing so little: a scoping review of interventions that address the stigma of mental illness in the Canadian context. Psychol Health Med. 2017; 22:507-23.

19. Earnshaw VA, Chaudoir SR. From conceptualizing to measuring HIV stigma: a review of HIV stigma mechanism measures. AIDS Behav. 2009;13:1160-77.

20. Mahajan AP, Sayles JN, Patel VA, Remien RH, Ortiz D, Szekeres G, et al. Stigma in the HIV/AIDS epidemic: a review of the literature and recommendations for the way forward. AIDS Lond Engl. 2008;22(Suppl 2): S67-79.

21. Loutfy M, Tharao W, Logie C, Aden MA, Chambers LA, Wu W, et al. Systematic review of stigma reducing interventions for African/black diasporic women. J Int AIDS Soc. 2015;18:19835

22. Monteiro SS, Villela WW, Soares PS. The interaction between axes of inequality in studies on discrimination, stigma and HIV/AIDS: contributions to the recent international literature. Glob Public Health. 2013;8:519-33.

23. Sandelowski M, Barroso J, Voils Cl. Gender, race/ethnicity, and social class in research reports on stigma in HIV-positive women. Health Care Women Int. 2009:30:273-88.

24. McCall L. The Complexity of Intersectionality. Signs. 2005;30:1771-800

25. Hancock A-M. When multiplication doesn't equal quick addition: examining intersectionality as a research paradigm. Perspect Polit. 2007;5:63-79.

26. Darlington CK, Hutson SP. Understanding HIV-related stigma among women in the southern United States: a literature review. AIDS Behav. 2017; 21:12-26.

27. Maulsby C, Millett G, Lindsey K, Kelley R, Johnson K, Montoya D, et al. HIV among black men who have sex with men (MSM) in the United States: a review of the literature. AIDS Behav. 2014;18:10-25.

28. Hatzenbuehler ML, Phelan JC, Link BG. Stigma as a fundamental cause of population health inequalities. Am J Public Health. 2013;103:813-21.

29. Corrigan PW, Markowitz FE, Watson AC. Structural levels of mental illness stigma and discrimination. Schizophr Bull. 2004;30:481-91.

30. Livingston JD, Boyd JE. Correlates and consequences of internalized stigma for people living with mental illness: a systematic review and meta-analysis. Soc Sci Med. 2010;71:2150-61.

31. Flanders L. No single-issue politics, only intersectionality: An interview with Kimberlé Crenshaw. Truthout. 2017; Available from:http://www.truth-out. org/opinion/item/40498-no-single-issue-politics-only-intersectionality-aninterview-with-kimberle-crenshaw. Accessed 22 Mar 2018

32. Abiri S, Oakley LD, Hitchcock ME, Hall A. Stigma related avoidance in people living with severe mental illness (SMI): findings of an integrative review. Community Ment Health J. 2016:52:251-61.

33. Ali K, Farrer L, Fassnacht DB, Gulliver A, Bauer S, Griffiths KM. Perceived barriers and facilitators towards help-seeking for eating disorders: a systematic review. Int J Eat Disord. 2017;50:9-21.

34. Ando S, Yamaguchi S, Aoki Y, Thornicroft G. Review of mental-health-related stigma in Japan. Psychiatry Clin Neurosci. 2013;67:471-82.

35. Ando S, Clement S, Barley EA, Thornicroft G. The simulation of hallucinations to reduce the stigma of schizophrenia: a systematic review. Schizophr Res. 2011;133:8-16.

36. Angermeyer MC, Holzinger A, Carta MG, Schomerus G. Biogenetic explanations and public acceptance of mental illness: systematic review of population studies. [review]. J Psychiatry. 2011:199(5):367-72.

37. Boyd JE, Adler EP, Otilingam PG, Peters T. Internalized stigma of mental illness (ISMI) scale: a multinational review. Compr Psychiatry, 2014:55:221-31.

38. Brohan E, Slade M, Clement S, Thornicroft G. Experiences of mental illness stigma, prejudice and discrimination: a review of measures. BMC Health Serv Res. 2010;10:80. 
39. Castaldelli-Maia JM, Scomparini LB, de Andrade AG, Bhugra D, de Toledo Ferraz Alves TC, D'Elia G. Perceptions of and attitudes toward antidepressants: stigma attached to their use-a review. J Nerv Ment Dis. 2011;199:866-71.

40. Clarke D, Usick R, Sanderson A, Giles-Smith L, Baker J. Emergency department staff attitudes towards mental health consumers: a literature review and thematic content analysis. Int J Ment Health Nurs. 2014;23(3):273-84

41. Clement S, Schauman O, Graham T, Maggioni F, Evans-Lacko S, Bezborodovs $\mathrm{N}$, et al. What is the impact of mental health-related stigma on help-seeking? A systematic review of quantitative and qualitative studies. Psychol Med. 2015:45:11-27.

42. Clement S, Lassman F, Barley E, EvansLacko S, Williams P, Yamaguchi S, et al. Mass media interventions for reducing mental health-related stigma [systematic review]. Cochrane Database Syst Rev. 2013; https://doi.org/10. 1002/14651858.CD009453.pub2.

43. Corrigan P, Michaels PJ, Morris S. Do the effects of antistigma programs persist over time? Findings from a meta-analysis. Psychiatr Serv. 2015;66: 543-6.

44. Corrigan PW, Morris SB, Michaels PJ, Rafacz JD, Rusch N. Challenging the public stigma of mental illness: a meta-analysis of outcome studies. Psychiatr Serv. 2012;63:963-73.

45. Dalky HF. Mental illness stigma reduction interventions: review of intervention trials. J Nurs Res. 2012;34:520-47.

46. Mendonça Lima CA, Lopes EST. Systematic review on origin of stigma and discrimination against old persons with mental disorders. Psychogeriatria Pol. 2012;9:87-106

47. Doley JR, Hart LM, Stukas AA, Petrovic K, Bouguettaya A, Paxton SJ. Interventions to reduce the stigma of eating disorders: a systematic review and meta-analysis. Int J Eat Disord. 2017;50:210-30.

48. Edwards D, Evans N, Gillen E, Longo M, Pryjmachuk S, Trainor G, et al. What do we know about the risks for young people moving into, through and out of inpatient mental health care? Findings from an evidence synthesis. Child Adolesc Psychiatry Ment Health. 2015;9:55.

49. Ellison N, Mason O, Scior K. Bipolar disorder and stigma: a systematic review of the literature. J Affect Disord. 2013;151:805-20.

50. Evans-Lacko S, Courtin E, Fiorillo A, Knapp M, Luciano M, Park A-L, et al. The state of the art in European research on reducing social exclusion and stigma related to mental health: a systematic mapping of the literature. [review]. J Assoc Eur Psychiatr. 2014;29:381-9.

51. Firmin RL, Luther L, Lysaker PH, Minor KS, Salyers MP. Stigma resistance is positively associated with psychiatric and psychosocial outcomes: a metaanalysis. Schizophr Res. 2016;175:118-28.

52. Gerlinger $\mathrm{G}$, Hauser M, De Hert M, Lacluyse K, Wampers M, Correll CU. Personal stigma in schizophrenia spectrum disorders: a systematic review of prevalence rates, correlates, impact and interventions. World Psychiatry. 2013;12:155-64.

53. Griffiths KM, Carron-Arthur B, Parsons A, Reid R. Effectiveness of programs for reducing the stigma associated with mental disorders. A meta-analysis of randomized controlled trials. World Psychiatry. 2014;13:161-75.

54. Hanisch SE, Twomey CD, Szeto ACH, Birner UW, Nowak D, Sabariego C. The effectiveness of interventions targeting the stigma of mental illness at the workplace: a systematic review. BMC Psychiatry. 2016;16:1.

55. Haugen PT, McCrillis AM, Smid GE, Nijdam MJ. Mental health stigma and barriers to mental health care for first responders: a systematic review and meta-analysis. J Psychiatr Res. 2017;94:218-29.

56. Hawke LD, Parikh SV, Michalak EE. Stigma and bipolar disorder: a review of the literature. J Affect Disord. 2013;150:181-91.

57. Janoušková M, Tušková E, Weissová A, Trančík P, Pasz J, Evans-Lacko S, et al. Can video interventions be used to effectively destigmatize mental illness among young people? A systematic review. Eur Psychiatry. 2017;41:1-9.

58. Jorm AF, Reavley NJ, Ross AM. Belief in the dangerousness of people with mental disorders: a review. J Psychiatry. 2012;46:1029-45.

59. Kaushik A, Kostaki E, Kyriakopoulos M. The stigma of mental illness in children and adolescents: a systematic review. Psychiatry Res. 2016;243:469-94.

60. Kvaale EP, Gottdiener WH, Haslam N. Biogenetic explanations and stigma: a meta-analytic review of associations among laypeople. Soc Sci Med. 2013; 96:95-103.

61. Kvaale EP, Haslam N, Gottdiener WH. The "side effects" of medicalization: a meta-analytic review of how biogenetic explanations affect stigma. Clin Psychol Rev. 2013;33:782-94.
62. Mak WWS, Poon CYM, Pun LYK, Cheung SF. Meta-analysis of stigma and mental health. Soc Sci Med. 2007:65:245-61.

63. Malachowski C, Kirsh B. Workplace antistigma initiatives: a scoping study. Psychiatr Serv. 2013;64:694-702.

64. Mascayano F, Tapia T, Schilling S, Alvarado R, Tapia E, Lips W, et al. Stigma toward mental illness in Latin America and the Caribbean: a systematic review. Rev Bras Psiquiatr. 2016;38:73-85.

65. Mehta N, Clement S, Marcus E, Stona A-C, Bezborodovs N, Evans-Lacko S, et al. Evidence for effective interventions to reduce mental health-related stigma and discrimination in the medium and long term: systematic review. Br J Psychiatry J Ment Sci. 2015;207:377-84.

66. Mestdagh A, Hansen B. Stigma in patients with schizophrenia receiving community mental health care: a review of qualitative studies. Soc Psychiatry Psychiatr Epidemiol. 2014;49:79-87.

67. Milton AC, Mullan BA. Diagnosis telling in people with psychosis. Curr Opin Psychiatry. 2014;27:302-7.

68. Mittal D, Sullivan G, Chekuri L, Allee E, Corrigan PW. Empirical studies of selfstigma reduction strategies: a critical review of the literature. [review]. Psychiatr Serv. 2012;63:974-81.

69. Mueller J, Callanan MM, Greenwood K. Communications to children about mental illness and their role in stigma development: an integrative review. J Ment Health Abingdon Engl. 2016;25:62-70.

70. Parcesepe AM, Cabassa LJ. Public stigma of mental illness in the United States: a systematic literature review. Admin Pol Ment Health. 2013;40:384-99.

71. Putman S. Mental illness: diagnostic title or derogatory term? (attitudes towards mental illness) developing a learning resource for use within a clinical call Centre. A systematic literature review on attitudes towards mental illness. J Psychiatr Ment Health Nurs. 2008;15(8):684-93.

72. Read J, Haslam N, Sayce L, Davies E. Prejudice and schizophrenia: a review of the "mental illness is an illness like any other" approach. Acta Psychiatr Scand. 2006;114:303-18.

73. Schnyder N, Panczak R, Groth N, Schultze-Lutter F. Association between mental health-related stigma and active help-seeking: systematic review and meta-analysis. Br J Psychiatry J Ment Sci. 2017;210:261-8.

74. Schomerus G, Schwahn C, Holzinger A, Corrigan PW, Grabe HJ, Carta MG, et al. Evolution of public attitudes about mental illness: a systematic review and meta-analysis. Acta Psychiatr Scand. 2012;125:440-52.

75. Sharp M-L, Fear NT, Rona RJ, Wessely S, Greenberg N, Jones N, et al. Stigma as a barrier to seeking health care among military personnel with mental health problems. Epidemiol Rev. 2015;37:144-62.

76. Stubbs A. Reducing mental illness stigma in health care students and professionals: a review of the literature. Australas Psychiatry. 2014;22:579-84

77. Thornicroft G, Mehta N, Clement S, Evans-Lacko S, Doherty M, Rose D, et al. Evidence for effective interventions to reduce mental-health-related stigma and discrimination. Lancet. 2016;387:1123-32.

78. Tsang HWH, Ching SC, Tang KH, Lam HT, Law PYY, Wan CN. Therapeutic intervention for internalized stigma of severe mental illness: a systematic review and meta-analysis. Schizophr Res. 2016;173:45-53.

79. Turan JM, Nyblade L. HIV-related stigma as a barrier to achievement of global PMTCT and maternal health goals: a review of the evidence. AIDS Behav. 2013;17:2528-39.

80. Wittkowski A, McGrath LK, Peters S. Exploring psychosis and bipolar disorder in women: a critical review of the qualitative literature. BMC Psychiatry. 2014;14:281

81. Wood L, Byrne R, Varese F, Morrison AP. Psychosocial interventions for internalised stigma in people with a schizophrenia-spectrum diagnosis: a systematic narrative synthesis and meta-analysis. Schizophr Res. 2016; 176:291-303

82. Xu Z, Rüsch N, Huang F, Kösters M. Challenging mental health related stigma in China: systematic review and meta-analysis. I. Interventions among the general public. Psychiatry Res. 2017;255:449-56.

83. Yamaguchi S, Mino Y, Uddin S. Strategies and future attempts to reduce stigmatization and increase awareness of mental health problems among young people: a narrative review of educational interventions. Psychiatry Clin Neurosci. 2011;65:405-15.

84. Yamaguchi S, Wu S-I, Biswas M, Yate M, Aoki Y, Barley EA, et al. Effects of short-term interventions to reduce mental health-related stigma in university or college students. J Nerv Ment Dis. 2013;201:490-503.

85. Yamaguchi S, Mizuno M, Ojio Y, Sawada U, Matsunaga A, Ando S, et al. Associations between renaming schizophrenia and stigma-related outcomes: a systematic review. Psychiatry Clin Neurosci. 2017;71:347-62. 
86. Yang LH, Thornicroft G, Alvarado R, Vega E, Link BG. Recent advances in cross-cultural measurement in psychiatric epidemiology: utilizing "what matters most" to identify culture-specific aspects of stigma. J Epidemiol. 2014:43:494-510.

87. Campbell C, Skovdal M, Gibbs A. Creating social spaces to tackle AIDSrelated stigma: reviewing the role of church groups in sub-Saharan Africa. AIDS Behav. 2011;15:1204-19

88. Colombini M, Stockl H, Watts C, Zimmerman C, Agamasu E, Mayhew SH. Factors affecting adherence to short-course ARV prophylaxis for preventing mother-to-child transmission of HIV in sub-Saharan Africa: a review and lessons for future elimination. AIDS Care. 2014;26:914-26.

89. Dao A, Hirsch JS, Giang LM, Parker RG. Social science research on HIV in Vietnam: a critical review and future directions. Glob Public Health. 2013; 8(Suppl 1):S7-S29.

90. Florom-Smith AL, De Santis JP. Exploring the concept of HIV-related stigma. Nurs Forum. 2012;47(3):153-65. Wiley Periodicals, Inc

91. Gesesew HA, Tesfay Gebremedhin A, Demissie TD, Kerie MW, Sudhakar M, Mwanri L. Significant association between perceived HIV related stigma and late presentation for HIV/AIDS care in low and middle-income countries: a systematic review and meta-analysis. PLoS One. 2017;12:e0173928.

92. Ho S-S, Holloway A. The impact of HIV-related stigma on the lives of HIV-positive women: an integrated literature review. [review]. J Clin Nurs. 2016;25:8-19.

93. Katz IT, Ryu AE, Onuegbu AG, Psaros C, Weiser SD, Bangsberg DR, et al. Impact of HIV-related stigma on treatment adherence: systematic review and meta-synthesis. J Int AIDS Soc. 2013;16(Suppl 2):18640.

94. Logie C, Gadalla TM. Meta-analysis of health and demographic correlates of stigma towards people living with HIV. AIDS Care. 2009;21:742-53.

95. Lorenc T, Marrero-Guillamón I, Llewellyn A, Aggleton P, Cooper C, Lehmann A, et al. HIV testing among men who have sex with men (MSM): systematic review of qualitative evidence. Health Educ Res. 2011;26:834-46.

96. Mahajan AP, Sayles JN, Patel VA, Remien RH, Sawires SR, Ortiz DJ, et al. Stigma in the HIV/AIDS epidemic: a review of the literature and recommendations for the way forward. AIDS. 2008;22(Suppl 2):S67-79.

97. Mak WWS, Mo PKH, Ma GYK, Lam MYY. Meta-analysis and systematic review of studies on the effectiveness of HIV stigma reduction programs. Soc Sci Med. 2017;188:30-40.

98. McAteer Cl, Truong N-AT, Aluoch J, Deathe AR, Nyandiko WM, Marete I, et al. A systematic review of measures of HIV/AIDS stigma in paediatric HIVinfected and HIV-affected populations. J Int AIDS Soc. 2016;19:21204.

99. Mills E, Wilson K, Rachlis B, Griffith L, Wu P, Guyatt G, et al. Barriers to participation in HIV drug trials: a systematic review. Lancet Infect Dis. 2006;6:32-8.

100. Monjok E, Smesny A, Essien EJ. HIV/AIDS-related stigma and discrimination in Nigeria: review of research studies and future directions for prevention strategies. J Reprod Health. 2009;13:21-35.

101. Monteiro S, Villela WV, Knauth D. Discrimination, stigma, and AIDS: a review of academic literature produced in Brazil (2005-2010). Cad Saude Publica. 2012:28:170-6.

102. Paudel V, Baral KP. Women living with HIV/AIDS (WLHA), battling stigma, discrimination and denial and the role of support groups as a coping strategy: a review of literature. Reprod Health. 2015;12:53.

103. Prost A, Elford J, Imrie J, Petticrew M, Hart GJ. Social, behavioural, and intervention research among people of sub-Saharan African origin living with HIV in the UK and Europe: literature review and recommendations for intervention. AIDS Behav. 2008;12:170-94.

104. Roger KS, Mignone J, Kirkland S. Social aspects of HIV/AIDS and aging: a thematic review. Can J Aging Rev Can Vieil. 2013;32:298-306.

105. Sengupta S, Banks B, Jonas D, Miles MS, Smith GC. HIV interventions to reduce HIV/AIDS stigma: a systematic review. AIDS Behav. 2011;15:1075-87.

106. Smith R, Rossetto K, Peterson BL. A meta-analysis of disclosure of one's HIVpositive status, stigma and social support. AIDS Care. 2008;20:1266-75.

107. Sweeney SM, Vanable PA. The association of HIV-related stigma to HIV medication adherence: a systematic review and synthesis of the literature. AIDS Behav. 2016;20:29-50.

108. Talley AE, Ann Bettencourt B. A relationship-oriented model of HIV-related stigma derived from a review of the HIV-affected couples literature. AIDS Behav. 2010;14:72-86.

109. Weihs M, Meyer-Weitz A. Barriers to workplace HIV testing in South Africa: a systematic review of the literature. AIDS Care. 2016;28:495-9.

110. Wilson MC, Scior K. Attitudes towards individuals with disabilities as measured by the implicit association test: a literature review. Res Dev Disabil. 2014;35:294-321.
111. Zeldenryk LM, Gray M, Speare R, Gordon S, Melrose W. The emerging story of disability associated with lymphatic filariasis: a critical review. PLoS Negl Trop Dis. 2011;5:e1366.

112. Grant MJ, Booth A. A typology of reviews: an analysis of 14 review types and associated methodologies. Health Inf Libr J. 2009;26:91-108.

113. Torraco RJ. Writing integrative literature reviews: guidelines and examples. Hum Resour Dev Rev. 2005;4:356-67.

114. de Souza MT, da Silva MD, de Carvalho R. Integrative review: what is it? How to do it? Einstein São Paulo. 2010:8:102-6.

115. Atkins S, Lewin S, Smith H, Engel M, Fretheim A, Volmink J. Conducting a meta-ethnography of qualitative literature: lessons learnt. BMC Med Res Methodol. 2008;8:21

116. Erwin EJ, Brotherson MJ, Summers JA. Understanding qualitative metasynthesis: issues and opportunities in early childhood intervention research. J Early Interv. 2011;33:186-200.

117. Paterson B, Thorne S, Canam C, Jillings C. Meta-study of qualitative health research. Thousand Oak: SAGE Publications, Inc.; 2001. https://doi.org/10. 4135/9781412985017.
Ready to submit your research? Choose BMC and benefit from:

- fast, convenient online submission

- thorough peer review by experienced researchers in your field

- rapid publication on acceptance

- support for research data, including large and complex data types

- gold Open Access which fosters wider collaboration and increased citations

- maximum visibility for your research: over $100 \mathrm{M}$ website views per year

At $\mathrm{BMC}$, research is always in progress.

Learn more biomedcentral.com/submissions 